\title{
Light NMSSM Higgs bosons in SUSY cascade decays at the LHC
}

\author{
Oscar Stål and Georg Weiglein \\ Deutsches Elektronen-Synchrotron DESY, \\ Notkestraße 85, D-22607 Hamburg, Germany \\ E-mail: oscar.stal@desy.de, georg.weiglein@desy.de
}

ABSTRACT: An interesting feature of the next-to-minimal supersymmetric standard model (NMSSM) is that one or more Higgs bosons may be comparably light $\left(M_{H_{i}}<M_{Z}\right)$ without being in conflict with current experimental bounds. Due to a large singlet component, their direct production in standard channels at the Large Hadron Collider (LHC) is suppressed. We demonstrate that there are good prospects for observing such a light Higgs boson in decays of heavy neutralinos and charginos. We consider an example scenario with $20 \mathrm{GeV}<M_{H_{1}}<M_{Z}$ and show that a large fraction of the cascade decays of gluinos and squarks involves the production of at least one Higgs boson. Performing a Monte Carlo analysis at the level of fast detector simulation, it is demonstrated how the Higgs signal can be separated from the main backgrounds, giving access to the Yukawa coupling of the Higgs to bottom quarks. Analyzing the resulting $b \bar{b}$ mass spectrum could provide an opportunity for light Higgs boson discovery already with $5 \mathrm{fb}^{-1}$ of LHC data at $7 \mathrm{TeV}$.

Keywords: Higgs Physics, Beyond Standard Model, Supersymmetric Standard Model

ARXIV EPRINT: 1108.0595 


\section{Contents}

1 Introduction 1

2 The next-to-minimal supersymmetric standard model 3

2.1 The Higgs sector 3

2.2 The neutralino sector 5

2.3 The squark sector 6

2.4 Scenarios with light Higgs bosons 6

3 Higgs production in the light $H_{1}$ scenario $\quad 9$

3.1 Standard channels 9

$\begin{array}{ll}3.2 \text { SUSY cascades } & 10\end{array}$

$4 \quad$ LHC analysis $\quad 14$

$\begin{array}{lll}4.1 \text { Event generation } & 15\end{array}$

$\begin{array}{llr}4.2 \text { Backgrounds } & 16\end{array}$

$\begin{array}{lll}4.3 & \text { Event selection } & 16\end{array}$

$\begin{array}{lll}4.4 & \text { Results } & 20\end{array}$

5 Summary and conclusions $\quad 23$

\section{Introduction}

The precise nature of the Higgs mechanism thought to be responsible for electroweak symmetry breaking remains unknown. To discover and study the properties of one or more Higgs bosons is therefore a challenge - and one of the major objectives - for the experiments at the running Large Hadron Collider (LHC). Most of the Higgs boson search strategies to date are designed to probe the Higgs sector of the Standard Model (SM), or its minimal supersymmetric extension (MSSM) [1, 2].

There are several theoretically appealing arguments for weak-scale supersymmetry to be realized in nature: it solves the hierarchy problem of the SM Higgs mass, it enables gauge coupling unification, and with R-parity conservation it also provides a natural dark matter candidate. On the other hand, the realization of weak-scale supersymmetry in terms of the MSSM is not free of theoretical problems, such as the scale for the bilinear $\mu$-parameter entering the MSSM superpotential with positive mass dimension. This parameter has no natural values besides $M_{\mathrm{GUT}}$ or zero, while at the same time it must be close to the electroweak scale for a phenomenologically acceptable theory. To solve this problem in an elegant way the MSSM can be extended by a complex scalar singlet, giving the so-called next-to-minimal supersymmetric model (NMSSM). In this model an effective $\mu$-term of 
the right size can be generated dynamically from supersymmetry-breaking operators. For a general introduction to the NMSSM we refer to the recent reviews [3, 4].

The NMSSM is characterized by an enlarged Higgs and neutralino sector as compared to the MSSM, giving rise in particular to a richer Higgs phenomenology. While it is well known that in certain scenarios of the MSSM with complex parameters a light Higgs, with mass much below that of the $Z$ boson, is unexcluded by the searches at LEP [5] and the Tevatron [6] (see [7, 8] for recent reevaluations with improved theoretical predictions), such a scenario can occur even more generically in the NMSSM. In order to be compatible with the limits from the LEP Higgs searches, in particular the couplings of such a light Higgs state to gauge bosons must be heavily suppressed. As a consequence of the presence of a Higgs singlet, in the NMSSM such a situation happens whenever a light Higgs state has a sufficiently large singlet component.

The search for a heavier Higgs state with SM-like (or only moderately suppressed) couplings to gauge bosons is complicated in such a scenario by the fact that often the decay of this heavier Higgs state into a pair of lighter Higgses is kinematically open, giving rise to unusual decay signatures and to a large suppression of the standard search channels for a SM-like Higgs. It should be noted in this context that the observation of a decay of a heavier Higgs into a pair of lighter Higgses would provide an opportunity for gaining experimental access to triple-Higgs couplings, which are a crucial ingredient of electroweak symmetry breaking via the Higgs mechanism.

While in the NMSSM the case of a very light pseudo-scalar, $M_{A_{1}}<2 m_{b}$, has found considerable attention in the literature, in particular in the context of "ideal" Higgs scenarios [9-14], we will focus in the following on scenarios with a light CP-even boson with $20 \mathrm{GeV}<M_{H_{1}}<M_{Z}$. Within the MSSM the best known example of a light Higgs that is unexcluded by the present search limits is the "hole" in the coverage of the CPX benchmark scenario [15] for $M_{H_{1}} \approx 45 \mathrm{GeV}$ and moderate values of $\tan \beta$ [5], see [8] for a detailed discussion of the dependence of the unexcluded parameter region on the choice of the various MSSM parameters. It will be difficult to cover this parameter region with the standard search channels at the LHC [16-18]. Various other (non-standard) search channels have been proposed which may provide additional sensitivity in the quest to close this "CPX hole" [16, 19-26].

In our analysis we investigate the prospects for production of light CP-even Higgs bosons in the NMSSM through cascade decays of heavy SUSY particles at the LHC. Such an analysis, where Higgs bosons are produced in association with — or in decays of other states of new physics, is necessarily more model-dependent than the Higgs search in SM-like channels. On the other hand, investigating Higgs physics in conjunction with the production of other states of new physics offers additional experimental opportunities and may also be more realistic, in the sense that in order to extract a Higgs signal backgrounds both from SM-type and new physics processes have to be considered. In the case of the MSSM with real parameters, for a Higgs with a mass above the LEP limit for a SM Higgs of $114.4 \mathrm{GeV}$ [27], a detailed experimental study for Higgs boson production in a SUSY cascade has been carried out by the CMS Collaboration [28], involving a full detector simulation and event reconstruction. These results, obtained for the benchmark point 
LM5, cannot be directly translated to the case of searches for a Higgs boson with mass far below $M_{Z}$, since in the latter case the $b$ jets resulting from the Higgs decay tend to be softer. The present work demonstrates an extension of Higgs searches in SUSY cascades to the case $M_{H}<M_{Z}$.

Further phenomenological analyses of Higgs production in SUSY cascades in the MSSM with real parameters (and Higgs masses above the LEP limits) have been carried out in [29-31], with recent developments focusing on jet substructure techniques to identify highly boosted Higgs bosons and enhance the discovery significance [32, 33]. The case of a lower mass Higgs has been investigated in [23], and it has been pointed out that in the CPX scenario there is a significant rate for producing a light MSSM Higgs boson in SUSY cascades, but no simulation of signal and background events was performed. In the NMSSM, Higgs production in the decays of neutralinos and/or charginos has been considered in [34, 35], while decays of third generation sfermions were investigated in [36].

We generalize and extend the earlier investigations by calculating the sparticle decay modes in a general NMSSM setting and performing a Monte Carlo simulation of the signal and the dominant background to the level of fast detector simulation. A simple cut-based analysis is performed to demonstrate that signal and background can be resolved in the $b \bar{b}+$ jets channel. The observation of the Higgs decay in the $b \bar{b}$ final state would be of interest also as a direct manifestation of the Higgs Yukawa coupling.

The outline of our paper is as follows: the next section begins with a brief recapitulation of the NMSSM, presenting the scenario with a light CP-even Higgs boson in some detail. In section 3, we describe the production of squarks and gluinos at the LHC and their eventual decay into Higgs bosons through electroweak cascades involving neutralinos and charginos. Section 4 describes a phenomenological Monte Carlo analysis of these cascade processes and contains the main results of this work in terms of kinematic distributions demonstrating the separation of signal and background. The conclusions are presented in section 5 .

\section{The next-to-minimal supersymmetric standard model}

In this section we review briefly the elements of the NMSSM which differ from the MSSM. Our conventions for the other sectors - that remain unchanged when going to the NMSSM — follow those of $[37,38]$.

\subsection{The Higgs sector}

The $Z_{3}$-symmetric version of the NMSSM is given by the scale-invariant superpotential

$$
W^{\mathrm{NMSSM}}=Y_{u} \hat{Q}_{L} \cdot \hat{H}_{u} \hat{U}_{L}^{c}+Y_{d} \hat{Q}_{L} \cdot \hat{H}_{d} \hat{D}_{L}^{c}+Y_{e} \hat{L}_{L} \cdot \hat{H}_{d} \hat{E}_{L}^{c}+\lambda \hat{S} \hat{H}_{u} \cdot \hat{H}_{d}+\frac{1}{3} \kappa \hat{S}^{3},
$$

where $\hat{\Phi}$ denotes a chiral superfield with scalar component $\Phi$. The complex gauge singlet $\hat{S}$ is a new addition with respect to the MSSM. To have a complete phenomenological model the soft SUSY-breaking terms must also be specified. These are extended by couplings of 
the singlet field, giving new contributions to the scalar potential

$$
V^{\mathrm{NMSSM}}=V^{\mathrm{MSSM}}+m_{S}^{2} S^{2}+\lambda A_{\lambda} S H_{u} \cdot H_{d}+\frac{1}{3} \kappa A_{\kappa} S^{3} .
$$

The NMSSM Higgs potential, which is derived from the usual $F$-terms, $D$-terms and the soft-breaking potential given by Equation (2.2), allows for a minimum where the singlet develops a vacuum expectation value (vev) $v_{s}=\langle S\rangle$. This induces an effective bilinear term $\lambda\langle S\rangle H_{u} \cdot H_{d}$, thus providing a dynamical explanation for the $\mu$ parameter of the MSSM in terms of $\mu_{\text {eff }}=\lambda v_{s}$.

Electroweak symmetry breaking (EWSB) proceeds similarly to the MSSM, and the two Higgs doublets are expanded around the potential minimum according to

$$
H_{d}=\left(\begin{array}{c}
v_{d}+\frac{1}{\sqrt{2}}\left(\phi_{d}-\mathrm{i} \sigma_{d}\right) \\
-\phi_{d}^{-}
\end{array}\right), \quad H_{u}=\left(\begin{array}{c}
\phi_{u}^{+} \\
v_{u}+\frac{1}{\sqrt{2}}\left(\phi_{u}+\mathrm{i} \sigma_{u}\right)
\end{array}\right) .
$$

Equivalently, the singlet field has an expansion

$$
S=v_{s}+\frac{1}{\sqrt{2}}\left(\phi_{s}+\mathrm{i} \sigma_{s}\right) .
$$

Using the minimization conditions for the potential, the scalar mass parameters $m_{H_{u}}^{2}, m_{H_{d}}^{2}$, and $m_{S}^{2}$ can be traded for

$$
\begin{aligned}
M_{Z}^{2} & =g^{2} v^{2}=\frac{1}{2}\left(g_{1}^{2}+g_{2}^{2}\right) v^{2}, \\
\tan \beta & =v_{u} / v_{d}, \\
\mu_{\mathrm{eff}} & =\lambda v_{s},
\end{aligned}
$$

where the doublet vevs fulfill $v^{2} \equiv v_{u}^{2}+v_{d}^{2}=(174 \mathrm{GeV})^{2}$. Assuming a CP-invariant Higgs sector, all parameters are taken to be real. The number of parameters required to specify the tree-level Higgs sector is increased with respect to the MSSM (where $\tan \beta$ and $M_{A}$ (or $M_{H^{ \pm}}$) are sufficient). In the NMSSM case we choose $\tan \beta, \lambda, \kappa, A_{\lambda}$, and $A_{\kappa}$ as the parameters describing the tree-level Higgs sector. We also use $\mu_{\text {eff }}$ as an input parameter rather than $v_{s}$ - to maintain the close correspondence to the $\mu$ parameter in the NMSSM.

After EWSB, the addition of a complex scalar field gives rise to additional particles in the NMSSM spectrum with respect to the MSSM: two additional Higgs bosons (one of which is CP-even, the other CP-odd) and their fermionic partner, the singlino. The elements of the tree-level mass matrix $\mathcal{M}_{H}^{2}$ for the CP-even Higgs bosons are given in the basis $\left(\phi_{d}, \phi_{u}, \phi_{s}\right)$ by

$$
\begin{aligned}
& \left(\mathcal{M}_{H}^{2}\right)_{11}=M_{Z}^{2} \cos ^{2} \beta+B \mu_{\mathrm{eff}} \tan \beta \\
& \left(\mathcal{M}_{H}^{2}\right)_{22}=M_{Z}^{2} \sin ^{2} \beta+B \mu_{\mathrm{eff}} \cot \beta \\
& \left(\mathcal{M}_{H}^{2}\right)_{33}=\frac{\lambda^{2} v^{2} A_{\lambda}}{\mu_{\mathrm{eff}}} \cos \beta \sin \beta+K \mu_{\mathrm{eff}}\left(A_{\kappa}+4 K \mu_{\mathrm{eff}}\right), \\
& \left(\mathcal{M}_{H}^{2}\right)_{12}=\left(2 \lambda^{2} v^{2}-M_{Z}^{2}\right) \cos \beta \sin \beta-B \mu_{\mathrm{eff}} \\
& \left(\mathcal{M}_{H}^{2}\right)_{13}=\lambda v\left[2 \mu_{\mathrm{eff}} \cos \beta-\left(B+K \mu_{\mathrm{eff}}\right) \sin \beta\right] \\
& \left(\mathcal{M}_{H}^{2}\right)_{23}=\lambda v\left[2 \mu_{\mathrm{eff}} \sin \beta-\left(B+K \mu_{\mathrm{eff}}\right) \cos \beta\right]
\end{aligned}
$$


where $B \equiv A_{\lambda}+K \mu_{\text {eff }}$ and $K \equiv \kappa / \lambda$. This matrix is diagonalized by a real $3 \times 3$ matrix with elements $S_{i j}$, such that the Higgs mass eigenstates $H_{i}$ are given by $H_{i}=S_{i j} \phi_{j}$. For the CP-odd states, the mass matrix elements in the basis $\left(\sigma_{d}, \sigma_{u}, \sigma_{s}\right)$ can be written

$$
\begin{aligned}
& \left(\mathcal{M}_{A}^{2}\right)_{11}=B \mu_{\mathrm{eff}} \tan \beta \\
& \left(\mathcal{M}_{A}^{2}\right)_{22}=B \mu_{\mathrm{eff}} \cot \beta \\
& \left(\mathcal{M}_{A}^{2}\right)_{33}=\frac{\lambda^{2} v^{2}}{\mu_{\mathrm{eff}}}\left(B+3 K \mu_{\mathrm{eff}}\right) \cos \beta \sin \beta-3 A_{\kappa} K \mu_{\mathrm{eff}}, \\
& \left(\mathcal{M}_{A}^{2}\right)_{12}=B \mu_{\mathrm{eff}} \\
& \left(\mathcal{M}_{A}^{2}\right)_{13}=\lambda v\left(B-3 K \mu_{\mathrm{eff}}\right) \sin \beta \\
& \left(\mathcal{M}_{A}^{2}\right)_{23}=\lambda v\left(B-3 K \mu_{\mathrm{eff}}\right) \cos \beta .
\end{aligned}
$$

Similarly to the CP-even case, the massive eigenstates $A_{i}$ can be written using a mixing matrix $P_{i j}$ as $A_{i}=P_{i j} \sigma_{j}$. One degree of freedom is massless and corresponds to the neutral Goldstone boson providing the longitudinal degree of freedom to the $Z$ boson. For some purposes it can be convenient to introduce the CP-odd mass parameter

$$
M_{A}^{2}=\frac{B \mu_{\mathrm{eff}}}{\cos \beta \sin \beta},
$$

which corresponds to the mass of the CP-odd Higgs boson in the MSSM limit of the NMSSM. ${ }^{1}$

No additional charged scalar is introduced in the NMSSM, but the relation of the physical charged Higgs boson mass to the CP-odd mass parameter gets modified. At tree-level the charged Higgs mass is now given by

$$
M_{H^{ \pm}}^{2}=M_{A}^{2}+M_{W}^{2}-\lambda^{2} v^{2} .
$$

\subsection{The neutralino sector}

As a result of introducing the new singlet superfield $\hat{S}$, the NMSSM comes with an additional fermion partner of the complex scalar $S$, the singlino. The singlino mixes with the existing four neutralinos of the MSSM. The resulting $5 \times 5$ mass matrix derives from the bilinear terms

$$
\mathcal{L}=-\frac{1}{2}\left(\tilde{\psi}^{0}\right)^{T} \mathcal{M}_{\tilde{\chi}^{0}} \tilde{\psi}^{0}+\text { h.c. }
$$

and in the basis $\left(-\mathrm{i} \tilde{B},-\mathrm{i} \tilde{W}, \tilde{H}_{d}, \tilde{H}_{u}, \tilde{S}\right)$ it is given by

$$
\mathcal{M}_{\tilde{\chi}^{0}}=\left(\begin{array}{ccccc}
M_{1} & 0 & -\frac{g_{1} v_{d}}{\sqrt{2}} & \frac{g_{1} v_{u}}{\sqrt{2}} & 0 \\
0 & M_{2} & \frac{g_{2} v_{d}}{\sqrt{2}} & -\frac{g_{2} v_{u}}{\sqrt{2}} & 0 \\
-\frac{g_{1} v_{d}}{\sqrt{2}} & \frac{g_{2} v_{d}}{\sqrt{2}} & 0 & -\mu_{\mathrm{eff}} & -\lambda v_{u} \\
\frac{g_{1} v_{u}}{\sqrt{2}} & -\frac{g_{2} v_{u}}{\sqrt{2}} & -\mu_{\mathrm{eff}} & 0 & -\lambda v_{d} \\
0 & 0 & -\lambda v_{u} & -\lambda v_{d} & 2 \kappa v_{s}
\end{array}\right) .
$$

\footnotetext{
${ }^{1}$ The MSSM limit is obtained by taking $\lambda \rightarrow 0, \kappa \rightarrow 0$, while keeping the ratio $K=\kappa / \lambda$ and all dimensionful parameters fixed.
} 
The upper left $4 \times 4$ submatrix is identical to the neutralino mass matrix in the MSSM. The neutralino masses can be diagonalized by a single unitary matrix $N$ such that

$$
D=\operatorname{diag}\left(m_{\tilde{\chi}_{i}^{0}}\right)=N^{*} \mathcal{M}_{\tilde{\chi}^{0}} N^{\dagger}
$$

is real and positive with the neutralino mass eigenvalues in ascending order. Alternatively one can use a real mixing matrix $N$, and allow $D$ to have negative elements. In this case the physical neutralino masses are given by $\left|m_{\tilde{\chi}_{i}^{0}}\right|$ and the neutralino couplings incorporate the additional phase shift on the neutralino fields.

\subsection{The squark sector}

We adopt a universal value $M_{\text {SUSY }}$ for the soft SUSY-breaking scalar mass parameters. This means that, for each squark pair $\tilde{q}_{L}, \tilde{q}_{R}$ of a given flavour, the mass matrix attains the form

$$
\mathcal{M}_{\tilde{q}}^{2}=\left(\begin{array}{cc}
M_{\mathrm{SUSY}}^{2}+m_{q}^{2}+M_{Z}^{2} \cos 2 \beta\left(I_{3}^{q}-Q_{q} s_{W}^{2}\right) & m_{q} X_{q} \\
m_{q} X_{q} & M_{\mathrm{SUSY}}^{2}+m_{q}^{2}+M_{Z}^{2} \cos 2 \beta Q_{q} s_{W}^{2}
\end{array}\right) .
$$

Here $m_{q}$ is the mass of the corresponding quark, $I_{3}^{q}$ the third component of the weak isospin, and $Q_{q}$ the electric charge quantum number. For the weak mixing angle we introduce the short-hand notations $s_{W} \equiv \sin \theta_{W}$ and $c_{W} \equiv \cos \theta_{W}$. The off-diagonal elements of $\mathcal{M}_{\tilde{q}}^{2}$ are related to the soft trilinear couplings $A_{q}$ as $X_{q}=A_{q}-\mu_{\text {eff }} \cot \beta$ for up-type squarks, and $X_{q}=A_{q}-\mu_{\text {eff }} \tan \beta$ for the case of down-type squarks, respectively. The mass eigenstates $\left(\tilde{q}_{1}, \tilde{q}_{2}\right)$ are obtained by a diagonalization of the mass matrix. A generic squark mass will be denoted $M_{\tilde{q}}$ below.

\subsection{Scenarios with light Higgs bosons}

As mentioned above, we will focus in the following on the case where the lightest CP-even Higgs boson of the NMSSM, $H_{1}$, has a mass much below $M_{Z}$. The fact that such a light Higgs, possessing a heavily suppressed coupling to gauge bosons as compared to the Higgs boson of the SM, may be unexcluded by the current search limits is known already from the case of the MSSM with complex parameters $[5,7,8]$. In the NMSSM such a situation happens more generically, in particular also for the case where the SUSY parameters are real. If the mass eigenstate $H_{1}$ has a large component of the singlet interaction state $\phi_{s}$, its couplings to gauge bosons (and also to quarks) will be correspondingly suppressed. We will investigate the prospects for detecting such a light Higgs state through its production in SUSY cascades.

In the numerical analysis, we shall use a scenario derived from the "P4" benchmark point defined in [39]. This benchmark can be realized in models with non-universal Higgs mass parameters $\left(m_{H_{u}} \neq m_{H_{d}}\right)$ at the scale of grand unification, and it is compatible with the data on the cold dark matter density. As originally defined, the $\mathrm{P} 4$ benchmark contains a very light CP-even Higgs boson $\left(M_{H_{1}}=32.3 \mathrm{GeV}\right)$. In order to explore the full range $M_{H_{1}}<M_{Z}$, we slightly modify the scenario to allow changing the value of $M_{H_{1}}$, with the remaining phenomenology essentially unchanged. To this end we set $\lambda=0.6$ and allow 


\begin{tabular}{|c|c|c|c|c|c|}
\hline \multicolumn{6}{|c|}{ Higgs sector parameters } \\
\hline$\lambda$ & 0.6 & & $\kappa$ & 0.12 & \\
\hline $\tan \beta$ & 2.6 & & $\mu_{\mathrm{eff}}$ & -200 & $\mathrm{GeV}$ \\
\hline$A_{\lambda}$ & -510 & $\mathrm{GeV}$ & $A_{\kappa}$ & $0-300$ & $\mathrm{GeV}$ \\
\hline \multicolumn{6}{|c|}{ Gaugino masses } \\
\hline$M_{1}$ & 300 & $\mathrm{GeV}$ & $M_{2}$ & 600 & $\mathrm{GeV}$ \\
\hline$M_{3}$ & 1000 & $\mathrm{GeV}$ & & & \\
\hline \multicolumn{6}{|c|}{$\begin{array}{c}\text { Trilinear couplings } \\
A_{t}=A_{b}=A_{\tau}=0 \mathrm{GeV}\end{array}$} \\
\hline \multicolumn{6}{|c|}{$\begin{array}{c}\text { Soft scalar mass } \\
M_{\mathrm{SUSY}}=750 \mathrm{GeV}, 1 \mathrm{TeV}\end{array}$} \\
\hline
\end{tabular}

Table 1. Values for the NMSSM input parameters at the SUSY-breaking scale in the modified P4 scenario.

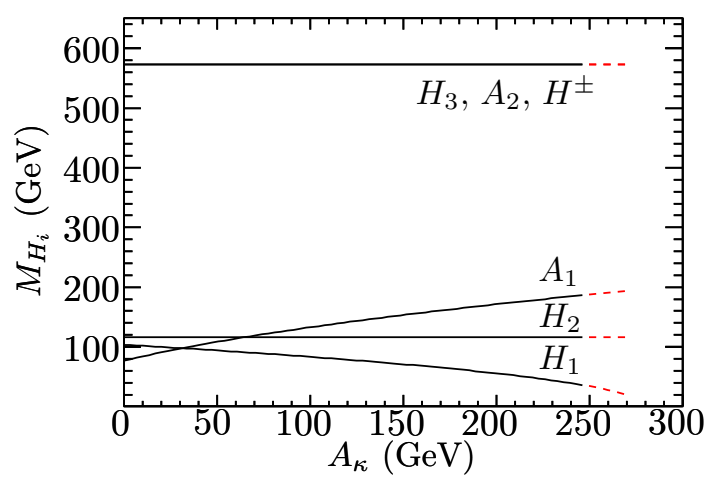

Figure 1. Higgs mass spectrum for the modified P4 scenario with $M_{\mathrm{SUSY}}=750 \mathrm{GeV}$ as a function of the free parameter $A_{\kappa}$. For $A_{\kappa} \gtrsim 250 \mathrm{GeV}$ (dashed) the electroweak symmetry remains unbroken in the global minimum.

$A_{\kappa}$ to take on values in the range $0 \mathrm{GeV}<A_{\kappa}<300 \mathrm{GeV} .^{2}$ The soft SUSY-breaking parameters are defined directly at the SUSY-breaking scale, allowing us to consider a more general spectrum for the remaining (non-Higgs) sectors of the theory. Values for the treelevel parameters in the Higgs sector and the soft SUSY-breaking parameters in the modified $\mathrm{P} 4$ scenario are specified in table 1 . The two values given for $M_{\mathrm{SUSY}}$ will be used later in the phenomenological analysis, while the values quoted in this and the next section (unless otherwise stated) have been evaluated for $M_{\mathrm{SUSY}}=750 \mathrm{GeV}$.

The NMSSM Higgs masses are subject to sizable corrections beyond leading order [40-45]. In order to incorporate the most accurate predictions currently available [46], NMSSMTools 2.3.5 [47-49] is used to compute the Higgs spectrum. The resulting Higgs masses in the modified $\mathrm{P} 4$ scenario are shown in figure 1 as a function of the free parameter $A_{\kappa}$. In the region with $A_{\kappa} \gtrsim 250 \mathrm{GeV}$ the global minimum of the Higgs potential does not break the electroweak symmetry; hence these values will not be considered. The masses of two Higgs bosons show a dependence on $A_{\kappa}$ : the lightest CP-even Higgs $H_{1}$

\footnotetext{
${ }^{2}$ The original P4 benchmark point is recovered for $\lambda=0.53$ and $A_{\kappa}=220 \mathrm{GeV}$.
} 
( $M_{H_{1}}$ varying from about $110 \mathrm{GeV}$ to $20 \mathrm{GeV}$ ), and the lightest CP-odd Higgs $A_{1}$ (with $M_{A_{1}}$ going from about $90 \mathrm{GeV}$ to $200 \mathrm{GeV}$ ). $H_{2}$ is always SM-like and has a mass of about $M_{H_{2}}=115 \mathrm{GeV}\left(118 \mathrm{GeV}\right.$ for $\left.M_{\mathrm{SUSY}}=1 \mathrm{TeV}\right)$. For all Higgs masses in this plot the NMSSM scenario is compatible with the direct limits from Higgs searches. The light CP-even Higgs $\left(M_{H_{1}} \ll M_{Z}\right)$ is allowed due to a large singlet component, with $\left|S_{13}\right|^{2}$ ranging from 0.9 for $A_{\kappa}=0 \mathrm{GeV}$ to $\left|S_{13}\right|^{2}>0.99$ for $A_{\kappa}=250 \mathrm{GeV}$. As a consequence, the couplings of $H_{1}$ to vector bosons are heavily suppressed, so that the cross section for production through Higgsstrahlung drops below the LEP limit. The pair production of $A_{1} H_{1}$ is even further suppressed by the large singlet fractions of both $H_{1}$ and $A_{1}$, while production of $H_{2} A_{1}$ and $H_{2} Z$ are beyond the kinematic reach of LEP. The full mass ranges shown in the figure are also compatible with the constraints from $B$-physics implemented in NMSSMTools 2.3.5 [47-49], as expected when the charged Higgs boson is heavy [50, 51]. The precise values obtained here for the heavy Higgs masses are $M_{H^{ \pm}} \simeq 563 \mathrm{GeV}$, and $M_{H_{3}} \simeq M_{A_{2}} \simeq 572 \mathrm{GeV}$. None of the heavy Higgs bosons will play any role in the following. With the negative sign for the effective $\mu$ parameter, this model cannot be used to explain the observed deviation in the anomalous magnetic moment of the muon (see e.g. [52] for a review). However - since the considered value of $\tan \beta$ is rather low - the predicted value for $(g-2)_{\mu}$ at least stays close to that in the SM.

The branching ratios of the three lightest Higgs states, $H_{1}, H_{2}$, and $A_{1}$, are given in figure 2. As can be seen from this figure, the light singlet $H_{1}$ decays preferentially into $b \bar{b}$, with $\operatorname{BR}\left(H_{1} \rightarrow b \bar{b}\right) \simeq 90 \%$ over the full mass range. The subdominant decay into $\tau \tau$ basically saturates the $H_{1}$ width. For lower values of $A_{\kappa}$ - where $M_{H_{1}} \gtrsim 90 \mathrm{GeV}$ - we note a similar enhancement of $\mathrm{BR}\left(H_{1} \rightarrow \gamma \gamma\right)$ compared to a SM Higgs with the same mass as recently discussed in [53]. The $H_{2}$ has a more complicated decay pattern, in particular for low $A_{\kappa}$ where $H_{2} \rightarrow b \bar{b}$ dominates and several competing modes $\left(H_{2} \rightarrow \tau \tau\right.$, $g g, W W$ ) each have a branching fraction around $10 \%$. In this region $H_{2}$ is SM-like, and the same search strategies as devised for the SM Higgs (and the lightest MSSM Higgs boson in the decoupling limit) should apply. This situation changes radically when the channel $H_{2} \rightarrow H_{1} H_{1}$ opens. When this is the case, the $H_{2} \rightarrow H_{1} H_{1}$ mode becomes completely dominant. Finally, the lightest CP-odd Higgs $A_{1}$ decays predominantly into $b \bar{b}$, with a large fraction going into the mode $A_{1} \rightarrow H_{1} Z$ when kinematically accessible.

In the neutralino sector the mass spectrum is independent of $A_{\kappa}$ (and $M_{\mathrm{SUSY}}$ ) at lowest order, cf. equation (2.10), and therefore remains fixed at:

$$
\begin{array}{ll}
M_{\tilde{\chi}_{1}^{0}}=97.6 \mathrm{GeV}, & M_{\tilde{\chi}_{2}^{0}}=227 \mathrm{GeV}, \quad M_{\tilde{\chi}_{3}^{0}}=228 \mathrm{GeV} \\
M_{\tilde{\chi}_{4}^{0}}=304 \mathrm{GeV}, & M_{\tilde{\chi}_{5}^{0}}=616 \mathrm{GeV} .
\end{array}
$$

There is a clear hierarchy in the mass parameters, which leads to a small mixing between the neutralinos. The heaviest neutralino is almost exclusively wino, and $\tilde{\chi}_{4}^{0}$ is mostly bino. The intermediate mass states $\tilde{\chi}_{2}^{0}$, and $\tilde{\chi}_{3}^{0}$ are predominantly Higgsino, while the lightest neutralino $\tilde{\chi}_{1}^{0}$ is the singlino. The lightest neutralino is also the overall lightest supersymmetric particle (LSP) in these scenarios and thereby a candidate for cold dark matter. 

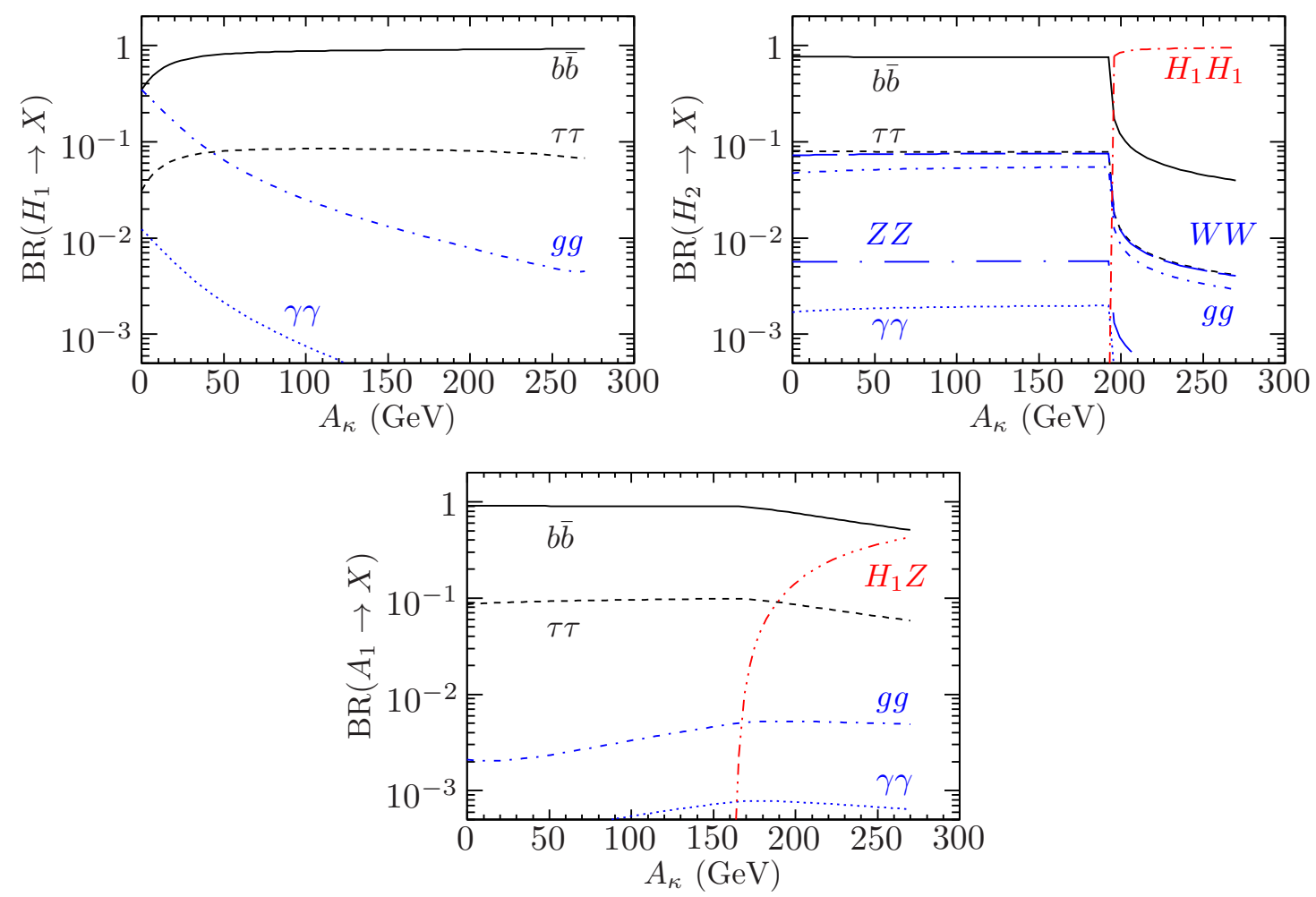

Figure 2. Branching ratios of $H_{1}$ (upper left), $H_{2}$ (upper right), and $A_{1}$ (lower) in the modified P4 scenario with $M_{\mathrm{SUSY}}=750 \mathrm{GeV}$.

\section{Higgs production in the light $H_{1}$ scenario}

\subsection{Standard channels}

The rate for direct production of a light singlet $H_{1}$ in gluon fusion, $g g \rightarrow H_{1}$, is proportional to its reduced (squared) coupling to quarks. Compared to a SM Higgs boson with the same mass, the dominant top loop contribution contains the additional factor $\left|S_{12}\right|^{2} / \sin ^{2} \beta$. The size of $\left|S_{12}\right|^{2}$ is limited from above by $\left|S_{12}\right|^{2} \leq 1-\left|S_{13}\right|^{2}$, where $S_{13}$ is the singlet component. For $M_{H_{1}} \ll M_{Z}$, where $\left|S_{13}\right| \rightarrow 1$, the rate for this process gets heavily suppressed. The cross section for $H_{1}$ in weak boson fusion, involving the coupling of $H_{1}$ to gauge bosons, is similarly suppressed. In scenarios where $M_{H_{1}}>M_{Z}$ (corresponding to the mass range below the LEP limit on a SM-like Higgs which is unexcluded in the MSSM with real parameters) the suppression of $g g \rightarrow H_{1}$ can be overcome by an increased branching ratio for $H_{1} \rightarrow \gamma \gamma$ [53].

For $A_{\kappa} \gtrsim 200 \mathrm{GeV}$ in the modified $\mathrm{P} 4$ scenario, $H_{1}$ is light enough to be produced through the decay of the SM-like $H_{2} \rightarrow H_{1} H_{1}$, see figure 2. The fact that this is an important (if not dominant) decay mode is a generic feature of scenarios where this kind of Higgs cascades is kinematically open. Since the production of $\mathrm{H}_{2}$ in standard channels is not suppressed, this could in principle be a very promising channel for discovery of $H_{2}, H_{1}$, or even both light Higgs bosons at the same time. However, the resulting two-step decay chain leads to "unusual" final states for $H_{2}: 4 b$ (about $82 \%$ of all decays), $2 b 2 \tau$ (17\%), and 
$4 \tau(0.6 \%)$, which makes it difficult to establish a Higgs signal. This has been demonstrated, for instance, by the numerous (unsuccessful) attempts [54-58] (see also [59]) to establish a "no-loose" theorem for NMSSM Higgs searches when decays of the SM-like Higgs into lighter Higgses are open.

Another possibility to produce $H_{1}$ in Higgs decays would be through the decay $A_{1} \rightarrow$ $H_{1} Z$. However, the singlet nature of $A_{1}$ in the modified $\mathrm{P} 4$ scenario leads to a suppression of $A_{1}$ production similar to that for $H_{1}$, and this mode is therefore not likely to be accessible.

The direct production of the heavy Higgs bosons $H_{3}, A_{2}$, and $H^{ \pm}$is in principle not suppressed with respect to the MSSM case, but at a mass close to $600 \mathrm{GeV}$ and low $\tan \beta$ the observation of those states at the LHC will be difficult even at high luminosity. A large fraction of the heavy Higgs bosons in this scenario will decay into lighter Higgs bosons, neutralinos and charginos. A detailed investigation of these channels could possibly be of interest for a study assuming a very high luminosity at $14 \mathrm{TeV}$, but is beyond the scope of the present paper.

In summary, it will be problematic to produce and reconstruct the light $H_{1}$ in any of the standard channels proposed for Higgs production at the LHC. We shall focus instead on the possibility to produce $H_{1}$ in the decays of supersymmetric particles.

\section{$3.2 \quad$ SUSY cascades}

As discussed in the previous section, inclusive production of the heavier state $H_{2}$ with subsequent decay $H_{2} \rightarrow H_{1} H_{1}$ may be difficult to observe at the LHC. However, the related process where a heavier neutralino decays into a lighter neutralino and a Higgs boson (and the corresponding decay mode of the heavier chargino) may offer better prospects. In fact, a light Higgs boson in the mass range below $M_{Z}$ may occur in a large fraction of cascade decays of heavier SUSY particles that are produced via strong interaction processes. The hard scale associated with the sparticle production can lead to event signatures which are more clearly separable from the SM backgrounds than those of inclusive Higgs production followed by a decay into a pair of $H_{1}$ states. The processes of interest are

$$
\begin{aligned}
\tilde{\chi}_{i}^{0} \rightarrow \tilde{\chi}_{j}^{0} H_{k}, & \tilde{\chi}_{i}^{0} \rightarrow \tilde{\chi}_{j}^{0} A_{k}, \\
\tilde{\chi}_{2}^{ \pm} \rightarrow \tilde{\chi}_{1}^{ \pm} H_{k}, & \tilde{\chi}_{2}^{ \pm} \rightarrow \tilde{\chi}_{1}^{ \pm} A_{k},
\end{aligned}
$$

where $H_{k}\left(A_{k}\right)$ denotes any of the CP-even (CP-odd) Higgs bosons. As mentioned above we do not consider scenarios where the heavier $H^{ \pm}$is produced in the cascades. The partial width for the neutralino decay (3.1) is given at tree-level by

$$
\Gamma\left(\tilde{\chi}_{i}^{0} \rightarrow \tilde{\chi}_{j}^{0} H_{k}\right)=\frac{\left|\mathcal{S}_{i j k}\right|^{2}}{16 \pi m_{\tilde{\chi}_{i}^{0}}^{3}} \tau^{1 / 2}\left(m_{\tilde{\chi}_{i}^{0}}^{2}, m_{\tilde{\chi}_{j}^{0}}^{2}, m_{H_{k}}^{2}\right)\left(m_{\tilde{\chi}_{i}^{0}}^{2}+m_{\tilde{\chi}_{j}^{0}}^{2}-m_{H_{k}}^{2}+2 m_{\tilde{\chi}_{i}^{0}} m_{\tilde{\chi}_{j}^{0}}\right),
$$

with a CP-even Higgs in the final state and

$$
\Gamma\left(\tilde{\chi}_{i}^{0} \rightarrow \tilde{\chi}_{j}^{0} A_{k}\right)=\frac{\left|\mathcal{P}_{i j k}\right|^{2}}{16 \pi m_{\tilde{\chi}_{i}^{0}}^{3}} \tau^{1 / 2}\left(m_{\tilde{\chi}_{i}^{0}}^{2}, m_{\tilde{\chi}_{j}^{0}}^{2}, m_{A_{k}}^{2}\right)\left(m_{\tilde{\chi}_{i}^{0}}^{2}+m_{\tilde{\chi}_{j}^{0}}^{2}-m_{A_{k}}^{2}-2 m_{\tilde{\chi}_{i}^{0}} m_{\tilde{\chi}_{j}^{0}}\right)
$$


for the decay into a CP-odd scalar. The Källén function $\tau(x, y, z)=(x-y-z)^{2}-4 y z$, and the coupling factors are

$$
\begin{aligned}
\mathcal{S}_{i j k}= & \frac{e}{2 c_{W} s_{W}}\left[\left(S_{k 1} N_{i 3}-S_{k 2} N_{i 4}\right)\left(c_{W} N_{j 2}-s_{W} N_{j 1}\right)\right] \\
& -\frac{\lambda}{\sqrt{2}}\left[N_{i 5}\left(S_{k 1} N_{j 4}+S_{k 2} N_{j 3}\right)+S_{k 3} N_{i 4} N_{j 3}\right]+\frac{\kappa}{\sqrt{2}} S_{k 3} N_{i 5} N_{j 5}+i \leftrightarrow j,
\end{aligned}
$$

and

$$
\begin{aligned}
-\mathrm{i} \mathcal{P}_{i j k}= & \frac{e}{2 c_{W} s_{W}}\left[\left(P_{k 1} N_{i 3}-P_{k 2} N_{i 4}\right)\left(c_{W} N_{j 2}-s_{W} N_{j 1}\right)\right] \\
& +\frac{\lambda}{\sqrt{2}}\left[N_{i 5}\left(P_{k 1} N_{j 4}+P_{k 2} N_{j 3}\right)+P_{k 3} N_{i 4} N_{j 3}\right]-\frac{\kappa}{\sqrt{2}} P_{k 3} N_{i 5} N_{j 5}+i \leftrightarrow j,
\end{aligned}
$$

where the mixing matrices $S_{i j}, P_{i j}$, and $N_{i j}$ are defined in section 2.1. Equations (3.3)(3.6) assume a real neutralino mixing matrix $N_{i j}$ and signed neutralino masses. In these expressions we can see explicitly the MSSM-like neutralino decays (proportional to the electromagnetic coupling $e$ ) and the NMSSM-specific contributions which are proportional to the Higgs-Higgsino coupling $\lambda$ and the singlet self-coupling $\kappa$. These terms both vanish in the MSSM-limit. Enhanced decays to a Higgs boson with a large singlet component can be expected from Higgsino-like neutralinos when $\lambda$ is large. As can also be seen from the similarity of the expressions above, there should be no large differences between the neutralino decay rates into $\mathrm{CP}$-even or CP-odd Higgs eigenstates (or CP-mixed states in the general case with $\mathrm{CP}$ violation). The neutralino decay modes competing with the Higgs modes are into vector bosons, $\tilde{\chi}_{i}^{0} \rightarrow \tilde{\chi}_{j}^{0} Z$ and $\tilde{\chi}_{i}^{0} \rightarrow \tilde{\chi}_{j}^{ \pm} W^{\mp}$. For brevity we refrain from giving expressions for these and the corresponding chargino decay modes here; they can be found in [34]. ${ }^{3}$ A detailed analysis of the $W^{ \pm}$mode is performed in [61]. Since the squarks and sleptons are assumed to be heavy, there are no open two-body decay modes of the neutralinos into the sfermion sector. Also slepton-mediated three-body decays - which can dominate over the two-body decays in certain scenarios - are numerically irrelevant for the same reason.

The branching fractions for the relevant decay channels have been computed at leading order using FeynArts/FormCalc $[62,63]$ and a purpose-built Fortran code. ${ }^{4}$ We have also compared our results to those from NMSDECAY [67] and find overall agreement. The neutralino branching ratios in the modified $\mathrm{P} 4$ scenario are shown in figure 3 . The decay modes of $\tilde{\chi}_{2}^{0}$ and $\tilde{\chi}_{3}^{0}$ (upper row of figure 3 ) — which are both Higgsino-like - show similar patterns for large values of $A_{\kappa}$. The dominant mode is always $\tilde{\chi}_{i}^{0} \rightarrow \tilde{\chi}_{1}^{0} Z$ with a branching ratio of about $50 \%$, but the Higgs channels are also significant with $\operatorname{BR}\left(\tilde{\chi}_{i}^{0} \rightarrow\right.$ $\left.\tilde{\chi}_{1}^{0} H_{1}\right) \gtrsim 0.3$ and $\operatorname{BR}\left(\tilde{\chi}_{i}^{0} \rightarrow \tilde{\chi}_{1}^{0} H_{2}\right) \sim 0.15$. An important point to note here is that the branching ratios of $\tilde{\chi}_{2}^{0}$ and $\tilde{\chi}_{3}^{0}$ are quite insensitive to changes in $M_{H_{1}}\left(A_{\kappa}\right)$. For the heavier neutralinos, $\tilde{\chi}_{4}^{0}$ and $\tilde{\chi}_{5}^{0}$ (lower row of figure 3 ), which also carry a larger gaugino fraction, the decay pattern is more complicated. Of largest interest for Higgs production is the sizable

\footnotetext{
${ }^{3}$ See also [60] for a discussion of neutralino signatures in singlet extensions of the MSSM.

${ }^{4}$ A FeynArts model file for the NMSSM has been obtained using FeynRules [64, 65] and SARAH [66]. Details on this implementation will be presented elsewhere.
} 

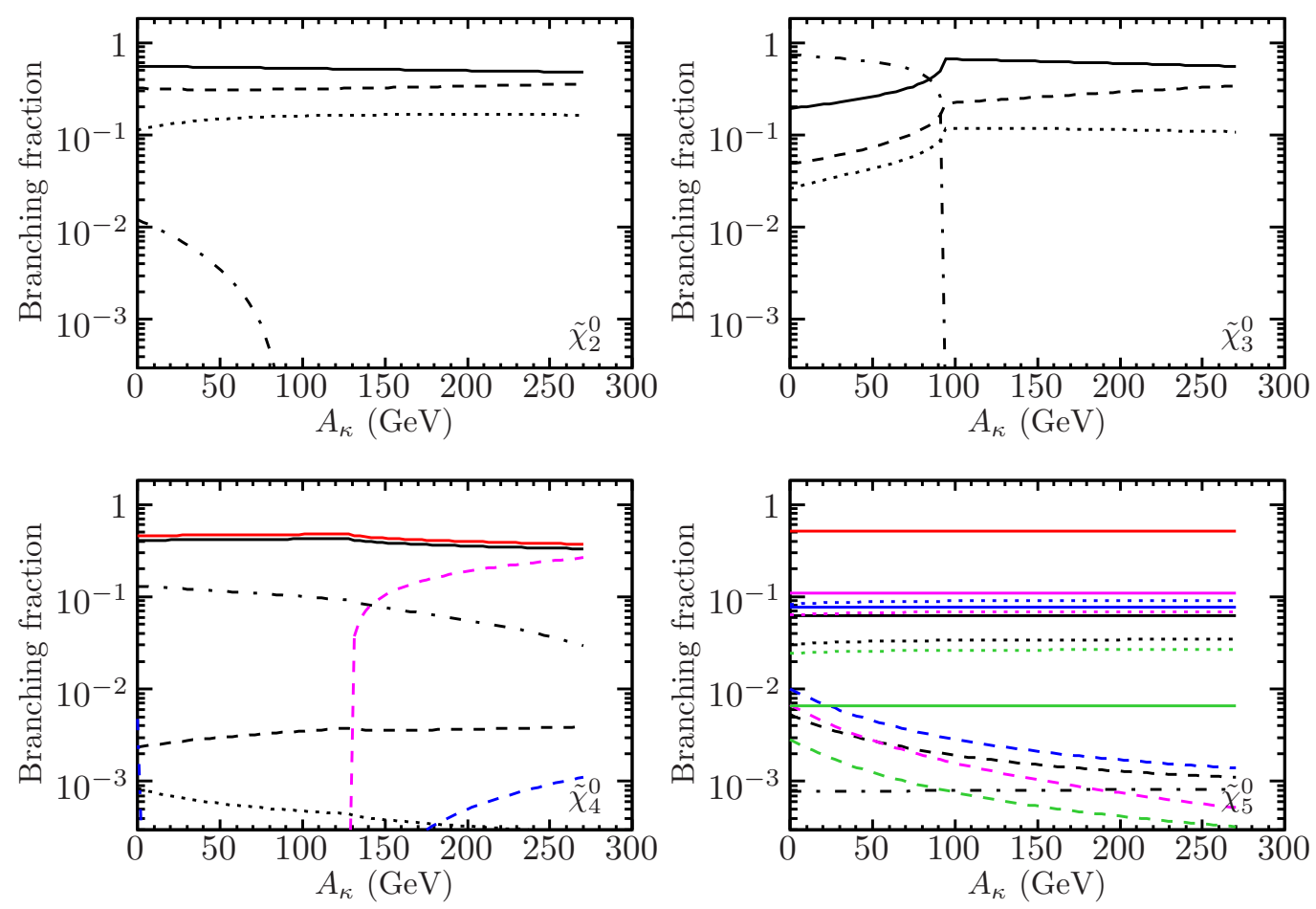

Figure 3. Branching ratios in the modified P4 scenario for $\tilde{\chi}_{i}^{0} \rightarrow \tilde{\chi}_{j}^{0} Z$ (solid), $\tilde{\chi}_{i}^{0} \rightarrow \tilde{\chi}_{j}^{0} H_{1}$ (dashed), $\tilde{\chi}_{i}^{0} \rightarrow \tilde{\chi}_{j}^{0} H_{2}$ (dotted), and $\tilde{\chi}_{i}^{0} \rightarrow \tilde{\chi}_{j}^{0} A_{1}$ (dot-dashed). The color coding indicates the final state neutralino $j=1$ (black), $j=2$ (blue), $j=3$ (magenta), $j=4$ (green), or the chargino mode $\tilde{\chi}_{i}^{0} \rightarrow \tilde{\chi}_{1}^{ \pm} W^{\mp}(\mathrm{red})$.

rate for $\tilde{\chi}_{4}^{0} \rightarrow \tilde{\chi}_{3}^{0} H_{1}$ (once $A_{\kappa}$ is sufficiently large to make this decay mode kinematically possible), and the fact that direct decays of $\tilde{\chi}_{5}^{0}$ to the LSP are suppressed. This will lead to neutralino decay chains with intermediate (Higgsino) steps. Everything taken together, we can expect a large number of light Higgs bosons to be produced in neutralino cascade decays.

The light chargino $\tilde{\chi}_{1}^{ \pm}$decays exclusively into the LSP and a $W$ boson, while the corresponding decay channels for the heavier chargino $\tilde{\chi}_{2}^{ \pm}$are shown in figure 4. Even if the dominant mode is $\tilde{\chi}_{2}^{ \pm} \rightarrow \tilde{\chi}_{1}^{ \pm} W^{\mp}$, independently of $A_{\kappa}$, there are several channels with a branching fraction of order $20 \%$ of interest for Higgs production. These include the mode $\tilde{\chi}_{2}^{ \pm} \rightarrow \tilde{\chi}_{1}^{ \pm} H_{2}$ and the decays into intermediate-mass Higgsinos, $\tilde{\chi}_{2}^{ \pm} \rightarrow \tilde{\chi}_{2,3}^{0} W^{ \pm}$.

The heavier neutralinos and the heavy chargino, from which a Higgs could emerge as decay product, can either be produced at the LHC directly or in the decay of a heavier SUSY particle. The cross section for direct production of neutralino pairs is small, only $\mathcal{O}(\mathrm{fb})$ at $\sqrt{s}=14 \mathrm{TeV}$, and the reach in these channels will be rather limited even for high luminosity. The large cross sections for production of strongly interacting sparticles (squarks and gluinos), on the other hand, are potentially more promising as a source of the heavier neutralino states and the heavier chargino. Exploiting cascade decays of this kind furthermore has the advantage that additional high- $p_{T}$ jets are produced, which facilitates triggering and event selection. 


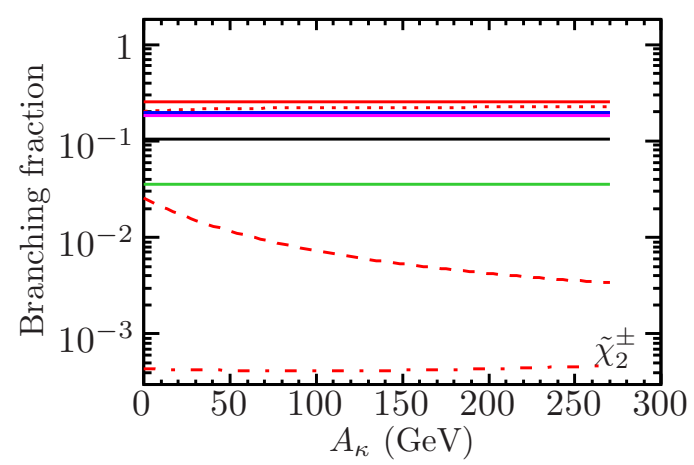

Figure 4. Branching ratios of $\tilde{\chi}_{2}^{ \pm}$in the modified P4 scenario. Neutralino final states $\tilde{\chi}_{2}^{ \pm} \rightarrow \tilde{\chi}_{i}^{0} W^{ \pm}$ (solid) are shown with the same color coding as for figure 3. Modes with a final state $\tilde{\chi}_{1}^{ \pm}$are shown in red: $\tilde{\chi}_{2}^{ \pm} \rightarrow \tilde{\chi}_{1}^{ \pm} Z$ (solid), $\tilde{\chi}_{2}^{ \pm} \rightarrow \tilde{\chi}_{1}^{ \pm} H_{1}$ (dashed), $\tilde{\chi}_{2}^{ \pm} \rightarrow \tilde{\chi}_{1}^{ \pm} H_{2}$ (dotted), $\tilde{\chi}_{2}^{ \pm} \rightarrow \tilde{\chi}_{1}^{ \pm} A_{1}$ (dot-dashed).

\begin{tabular}{|cc|ccccc|cccccc|}
\hline \multicolumn{10}{|c|}{ Masses $(\mathrm{GeV})$} & \multicolumn{8}{c|}{$\sigma_{\mathrm{LO}}(\mathrm{pb})$} & \multicolumn{1}{c|}{$\sigma_{\mathrm{NLO}}(\mathrm{pb})$} \\
$M_{\tilde{g}}$ & $M_{\tilde{q}}$ & $\tilde{g} \tilde{g}$ & $\tilde{q} \tilde{q}$ & $\tilde{g} \tilde{q}$ & $\tilde{q} \tilde{\bar{q}}$ & $\Sigma$ & $\tilde{g} \tilde{g}$ & $\tilde{q} \tilde{q}$ & $\tilde{g} \tilde{q}$ & $\tilde{q} \tilde{\bar{q}}$ & $\Sigma$ \\
\hline \multicolumn{10}{|c}{$\sqrt{s}=7 \mathrm{TeV}$} \\
\hline 750 & 750 & 0.03 & 0.23 & 0.25 & 0.05 & 0.56 & 0.07 & 0.27 & 0.39 & 0.08 & 0.82 \\
1000 & 750 & 0.002 & 0.19 & 0.06 & 0.05 & 0.31 & 0.006 & 0.21 & 0.10 & 0.07 & 0.39 \\
1000 & 1000 & 0.001 & 0.03 & 0.02 & 0.004 & 0.06 & 0.005 & 0.04 & 0.04 & 0.006 & 0.08 \\
\hline \multicolumn{10}{|c}{$\sqrt{s}=14 \mathrm{TeV}$} \\
\hline 750 & 750 & 1.18 & 1.67 & 5.20 & 1.06 & 9.11 & 2.21 & 2.06 & 6.78 & 1.53 & 12.6 \\
1000 & 750 & 0.15 & 1.41 & 1.86 & 0.96 & 4.38 & 0.32 & 1.59 & 2.44 & 1.34 & 5.69 \\
1000 & 1000 & 0.14 & 0.42 & 0.87 & 0.18 & 1.61 & 0.31 & 0.51 & 1.19 & 0.26 & 2.27 \\
1500 & 1500 & 0.01 & 0.04 & 0.05 & 0.01 & 0.10 & 0.01 & 0.05 & 0.07 & 0.02 & 0.15 \\
\hline
\end{tabular}

Table 2. Total production cross sections for $p p \rightarrow \tilde{g} \tilde{g}, p p \rightarrow \tilde{q} \tilde{q}, p p \rightarrow \tilde{g} \tilde{q}$, and $p p \rightarrow \tilde{q} \tilde{\bar{q}}$ at LO and NLO SUSY-QCD from Prospino. The squark cross sections are summed over the four "light" squark flavours. No kinematic cuts have been applied here.

We use Prospino to calculate the NLO cross sections for production of $p p \rightarrow \tilde{g} \tilde{g}$, $p p \rightarrow \tilde{q} \tilde{q}, p p \rightarrow \tilde{q} \tilde{\bar{q}}$, and $p p \rightarrow \tilde{g} \tilde{q}$ according to [68], with CTEQ6 [69] parton distributions and a common choice of renormalization and factorization scales as the average mass of the final state (SUSY) particles. Numerical results are given in table 2 for the two centre-ofmass energies $7 \mathrm{TeV}$ and $14 \mathrm{TeV}$. The cross sections for $p p \rightarrow \tilde{\bar{t}}$ and $p p \rightarrow \tilde{b} \tilde{\bar{b}}[70]$ are also calculated and included in the analysis, but since they turn out to be significantly smaller than $\sigma(p p \rightarrow \tilde{q} \tilde{\bar{q}})$ they are not shown in the table. In order to give some indication of the expected change in the number of events for different scenarios, the results are presented for several values of the squark masses, $M_{\tilde{q}}$, and the gluino mass, $M_{\tilde{g}}$. The mass ranges are selected to respect the published limits from ATLAS [71-75] and CMS [76-79] based on the 2010 data. Taking into account also the most recent results [80, 81], the $M_{\tilde{q}}=750 \mathrm{GeV}$ case appears to be under some pressure. We present the results of our analysis below for the two cases $M_{\mathrm{SUSY}}=750 \mathrm{GeV}$ and $M_{\mathrm{SUSY}}=1 \mathrm{TeV}$ (the leading order squark masses 


\begin{tabular}{|ccccc|}
\hline Final state & $\tilde{u}_{L}$ & $\tilde{u}_{R}$ & $\tilde{d}_{L}$ & $\tilde{d}_{R}$ \\
\hline$\tilde{q} \rightarrow q \tilde{\chi}_{1}^{0}$ & $2.9 \times 10^{-3}$ & $2.6 \times 10^{-3}$ & $6.3 \times 10^{-3}$ & $2.6 \times 10^{-3}$ \\
$\tilde{q} \rightarrow q \tilde{\chi}_{2}^{0}$ & $8.1 \times 10^{-3}$ & $5.4 \times 10^{-3}$ & $1.6 \times 10^{-2}$ & $5.4 \times 10^{-3}$ \\
$\tilde{q} \rightarrow q \tilde{\chi}_{3}^{0}$ & $1.9 \times 10^{-3}$ & $4.5 \times 10^{-2}$ & $2.0 \times 10^{-2}$ & $4.6 \times 10^{-2}$ \\
$\tilde{q} \rightarrow q \tilde{\chi}_{4}^{0}$ & $6.6 \times 10^{-2}$ & 0.95 & $2.9 \times 10^{-2}$ & 0.95 \\
$\tilde{q} \rightarrow q \tilde{\chi}_{5}^{0}$ & 0.29 & - & 0.32 & - \\
$\tilde{q} \rightarrow q^{\prime} \tilde{\chi}_{1}^{ \pm}$ & $9.6 \times 10^{-2}$ & - & $3.3 \times 10^{-4}$ & - \\
$\tilde{q} \rightarrow q^{\prime} \tilde{\chi}_{2}^{ \pm}$ & 0.54 & - & 0.61 & - \\
\hline
\end{tabular}

Table 3. Branching ratios for the first and second generation squarks into neutralinos and charginos in the modified P4 scenario with $M_{\mathrm{SUSY}}=750 \mathrm{GeV}$. Results for channels with a branching ratio below $10^{-4}$ are not shown.

are obtained from $M_{\text {SUSY }}$ through eq. (2.12), to which higher order corrections are then added).

The nearly mass-degenerate squarks decay preferentially into the SUSY-EW sector. Direct decays into Higgs bosons (or Higgsinos) are negligible for squarks of the first two generations due to the small Yukawa couplings. In contrast to the MSSM, the neutralinos also have a singlino component to which no squark couples. The left-handed squarks decay mainly into the wino, $\tilde{q}_{L} \rightarrow \tilde{W}^{0} q, \tilde{q}_{L} \rightarrow \tilde{W}^{ \pm} q^{\prime}$, while the right-handed squarks decay mostly to the bino, $\tilde{q}_{R} \rightarrow \tilde{B} q$. Numerically, this leads to squark decay modes listed in table 3 for the case with a soft scalar mass of $M_{\mathrm{SUSY}}=750 \mathrm{GeV}$. The squark decay pattern for $M_{\mathrm{SUSY}}=1 \mathrm{TeV}$ is qualitatively similar. ${ }^{5}$ Since the gaugino components are largest in the two heaviest neutralinos, the neutralinos produced in the squark decays tend to give rise to cascade decays with several steps.

Finally, we note that the gluinos decay 'democratically' through $\tilde{g} \rightarrow \tilde{q} \bar{q}$ into all flavours, with rates governed only by the available phase space.

\section{LHC analysis}

In order to assess whether the process discussed in the previous section can be useful as a Higgs search channel at the LHC we perform a Monte Carlo simulation. Here we use as benchmark the modified P4 scenario with the two different settings for the soft scalar mass: $M_{\mathrm{SUSY}}=750 \mathrm{GeV}$ and $M_{\mathrm{SUSY}}=1 \mathrm{TeV}$. The $\overline{\mathrm{DR}}$ value of the gluino mass parameter is set to $M_{3}=1 \mathrm{TeV}$. We select $A_{\kappa}$ such that $M_{H_{1}} \simeq 40 \mathrm{GeV}$, which also affects $M_{H_{2}}, M_{A_{1}}$ and the branching ratios in the two cases as discussed in section 3. We have chosen this value of $M_{H_{1}}$ as an illustrative example of our scenario with $20 \mathrm{GeV}<M_{H_{1}}<M_{Z}$ and in order to make contact with the analyses of the "CPX hole" in the MSSM with complex parameters. Our results however depend only very mildly on the specific choice for $M_{H_{1}}$. The simulation results are presented below both for LHC running at centre-of-mass energies of $7 \mathrm{TeV}$ and $14 \mathrm{TeV}$.

\footnotetext{
${ }^{5}$ The main numerical difference is an increase of $\operatorname{BR}\left(\tilde{u}_{L} \rightarrow q^{\prime} \tilde{\chi}_{2}^{ \pm}\right)$to $60 \%$ at the expense of a reduced $\operatorname{BR}\left(\tilde{u}_{L} \rightarrow q^{\prime} \tilde{\chi}_{1}^{ \pm}\right)=3.9 \times 10^{-2}$.
} 
The squark and gluino-induced cascades in general give rise to a final state with high multiplicities and several hard jets, as well as large missing transverse momentum due to the presence of the LSP at the end of each decay chain. The minimal signal cascades (defined to be those with at least one Higgs boson present) generated by the production of a single squark or gluino correspond to

$$
\begin{array}{lll}
\tilde{q} \rightarrow q \tilde{\chi}_{i}^{0} \rightarrow q \tilde{\chi}_{1}^{0} H_{k} \rightarrow q \tilde{\chi}_{1}^{0} b \bar{b}, & n_{j} \geq 1, & n_{b} \geq 2, \\
\tilde{g} \rightarrow g \tilde{q} \rightarrow g q \tilde{\chi}_{i}^{0} \rightarrow g q \tilde{\chi}_{1}^{0} H_{k} \rightarrow g q \tilde{\chi}_{1}^{0} b \bar{b}, & n_{j} \geq 2, & n_{b} \geq 2 .
\end{array}
$$

Equations (4.1a) and (4.1b) show the minimum number of light and heavy flavour (b-) jets expected in the signal. Each event contains production of a pair of sparticles and their associated jets, meaning that the full signature for production of at least one $H_{1}$ in the hadronic final state will be $n_{j} \geq 2, n_{b} \geq 2$. Since direct decays of the heavier (mainly gaugino) neutralinos into the singlino LSP are practically absent (cf. figure 3), most signal cascades will contain an intermediate Higgsino step which will add further particles in the final state. The typical jet multiplicity will also be higher due to additional QCD activity, in particular for gluon-initiated processes.

\subsection{Event generation}

For the event generation, we use MadGraph/MadEvent 4.4.44 [82] to calculate the leading order matrix elements for $p p \rightarrow \tilde{g} \tilde{g}, \tilde{q} \tilde{q}, \tilde{g} \tilde{q}, \tilde{q} \tilde{\bar{q}}, \tilde{t} \tilde{t}$, and $\tilde{b} \tilde{\bar{b}}$. The different event categories are weighted by the corresponding NLO cross sections to produce an inclusive SUSY sample. The resonance decay chains are then generated with PYTHIA 6.4 [83] using the NMSSM decay rates calculated above as input through the SUSY Les Houches accord [38]. The PYTHIA generator is also used to produce additional QCD radiation through initial- and final state parton showers (with $k_{T}$-ordered shower evolution), for parton fragmentation, and to generate multiple interactions for the underlying event. This produces fully dressed hadronic events which are passed through the fast simulation of the ATLAS detector performance implemented in the Delphes package [84]. ${ }^{6}$ Hadronic jets are clustered using the anti- $k_{T}$ algorithm [85] with a jet radius measure of $R=0.4$.

Since for the lightest Higgs boson the decay to $b \bar{b}$ is favored, the probability $\eta_{b}$ to correctly identify jets originating from bottom partons ( $b$-tagging efficiency) becomes a crucial quantity for the analysis. Based on [86] we parametrize this efficiency as a constant $\eta_{b}=0.6$ with respect to both the detector geometry and the jet energy scale. Only jets in the central tracking region $|\eta|<2.5$ can be tagged. The rate for misidentification as a $b$-jet is assumed to be $\eta_{c}=0.1$ for charm jets, and $\eta_{q}=0.01$ for jets produced by light quarks and gluons. The actual tagging algorithm implemented in the Delphes simulation is not based on a particular experimental method to identify $b$-jets. The algorithm determines if a jet is close enough in $\Delta R$ to a "true" $b$ parton. When this is the case, the efficiencies given above are applied to determine if the tagging is successful or not.

\footnotetext{
${ }^{6}$ Running the same Delphes analysis with the "CMS" detector setup and similar parameters for jets and heavy flavour tagging, no significant differences are observed in the output.
} 


\subsection{Backgrounds}

Based on the event signature, SM production of $t \bar{t}$ with at least one hadronically decaying $W$ boson (or additional jet activity) constitutes an irreducible background to the Higgs signal. We can a priori expect this to be the most important SM background since the scale for the SUSY-QCD processes is high $(>1 \mathrm{TeV})$. In principle there are other sources of background from production of $W+$ jets $(b \bar{b}), Z+$ jets $(b \bar{b})$, direct production of $b \bar{b}+$ jets, or from QCD multijets. The cross sections for these processes are large compared to the signal cross section, with QCD multijets the largest and thereby potentially the most serious. However, for QCD jet production to constitute a background to the Higgs signal simultaneously a double misidentification of heavy flavour jets and a large mismeasurement of the missing transverse energy is required. It is furthermore difficult to simulate this background reliably, since extreme kinematical fluctuations - or experimental effects would be necessary to produce the signal-like events. A detailed study of the experimental effects would require a full detector simulation, which is beyond the scope of the present paper. However, the dominance of the $t \bar{t}$ background over other SM processes, such as $W+$ jets or $Z+$ jets, for our final state has also been demonstrated experimentally by the results from SUSY searches with $b$-jets and missing $E_{T}$ [73]. We therefore proceed under the assumption that the cuts devised to suppress the irreducible $t \bar{t}$ background will also be efficient for suppressing the other SM backgrounds as well.

For the normalization of the $t \bar{t}$ background we use the NLO cross section $\sigma(p p \rightarrow$ $t \bar{t})=902 \mathrm{pb}(\sqrt{s}=14 \mathrm{TeV})$ and $\sigma(p p \rightarrow t \bar{t})=162 \mathrm{pb}(\sqrt{s}=7 \mathrm{TeV})$, computed with the HATHOR package [87] for $m_{t}=173.3 \mathrm{GeV}$ and MSTW2008 PDFs [88]. In this way a consistent NLO normalization is used for both the signal and background events. The $t \bar{t}$ background is generated in the same Monte Carlo framework as already described for the signal.

In addition to the SM backgrounds, the process we are interested in receives an important background from the SUSY cascade itself. Any final state containing two $b$-jets which do not result from an intermediate Higgs boson contributes to this background. Attempting to suppress the SUSY background events would require additional cuts that depend on the kinematics of the decay chains. This is something which may indeed be possible to devise once information on the supersymmetric spectrum has become available, but since we do not want to make any particular assumptions on the pattern of the SUSY spectrum, no selection will be applied aiming to reduce the SUSY background. Instead we will consider the inclusive $b \bar{b}$ mass spectrum directly after applying the cuts designed to reduce the SM background to determine if a Higgs signal can be extracted.

\subsection{Event selection}

As a first step, we perform a preselection of the expected event topology, demanding $n_{j} \geq 2$, $n_{b} \geq 2$. All reconstructed jets are required to have a minimum $p_{T} \geq 25 \mathrm{GeV}$.

Figure 5 shows the $p_{T}$ distribution for the hardest jet in each event, comparing the inclusive SUSY events (with $M_{\mathrm{SUSY}}=750 \mathrm{GeV}$ ) to the $t \bar{t}$ background. We show the results for the two cases $\sqrt{s}=7 \mathrm{TeV}$ (left) and $\sqrt{s}=14 \mathrm{TeV}$ (right). In order to illustrate the 

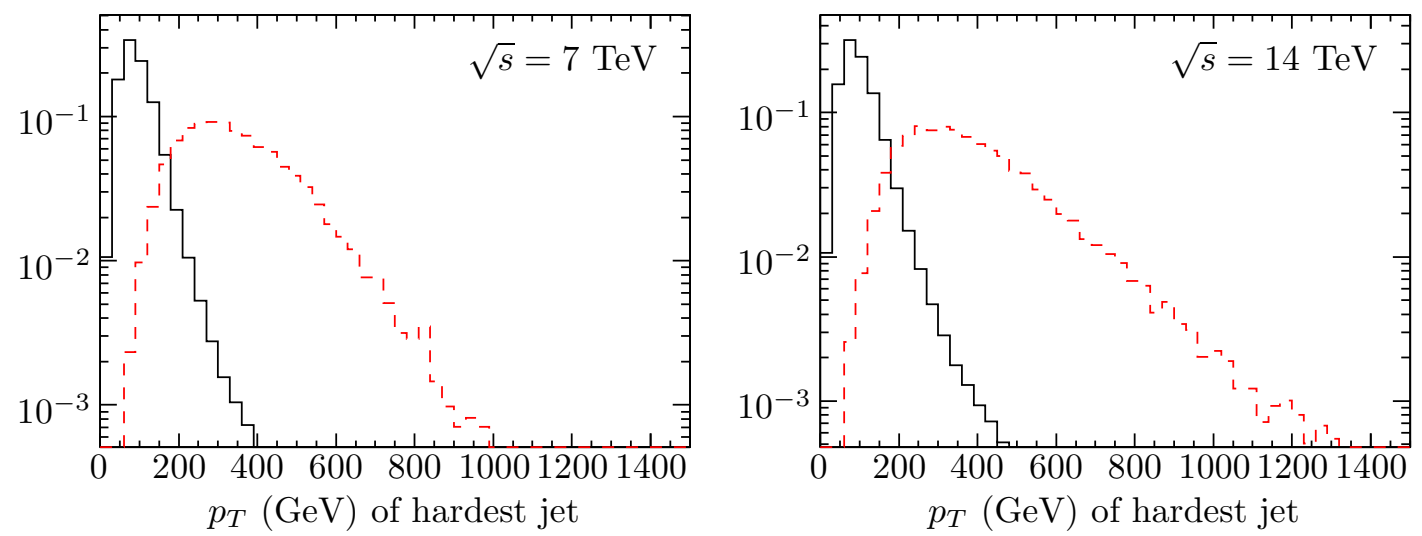

Figure 5. Transverse momentum distribution of the hardest (non-b) jet at $7 \mathrm{TeV}$ (left) and $14 \mathrm{TeV}$ (right) for the inclusive SUSY sample with $M_{\mathrm{SUSY}}=750 \mathrm{GeV}$ (dashed) and SM $t \bar{t}$ background (solid). The histograms are normalized to unity.
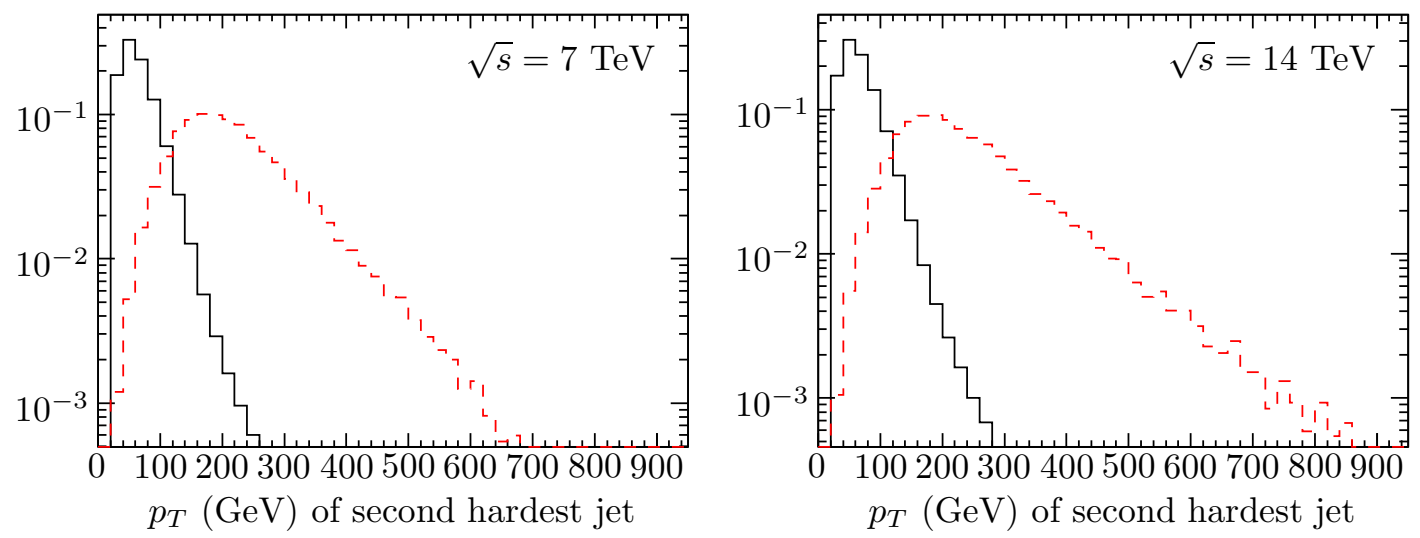

Figure 6. Transverse momentum distribution of the second hardest jet at $7 \mathrm{TeV}$ (left) and $14 \mathrm{TeV}$ (right) for the inclusive SUSY sample with $M_{\mathrm{SUSY}}=750 \mathrm{GeV}$ (dashed) and SM $t \bar{t}$ background (solid). The histograms are normalized to unity.

effect of applying cuts to this variable, each histogram is normalized to unity. From figure 5 it is clear that the leading jet from the SUSY events has a much harder scale compared to the $t \bar{t}$ events. This can be understood as a result of the large boost obtained by the light quark jets originating from squark decays. It can also be seen that there is only a minor scaling difference in the jet $p_{T}$ distribution between the $7 \mathrm{TeV}$ and $14 \mathrm{TeV}$ cases. The same is true for the second hardest (light) jet, for which the corresponding $p_{T}$ distribution is shown in figure 6. Similar differences between signal and background can be observed also for the third and fourth jet when they are present.

With each cascade ending in the stable LSP, a large missing transverse energy $\mathbb{E}_{T}$ is expected for the signal events. This distribution is displayed in figure 7 , and shows indeed that the SUSY distribution peaks at high $\mathbb{E}_{T}$ values $(\gtrsim 200 \mathrm{GeV})$. This is therefore an important discriminating variable to suppress the background from $t \bar{t}$ events, where the missing transverse energy is due to neutrinos from leptonic $W$ decays. As already 

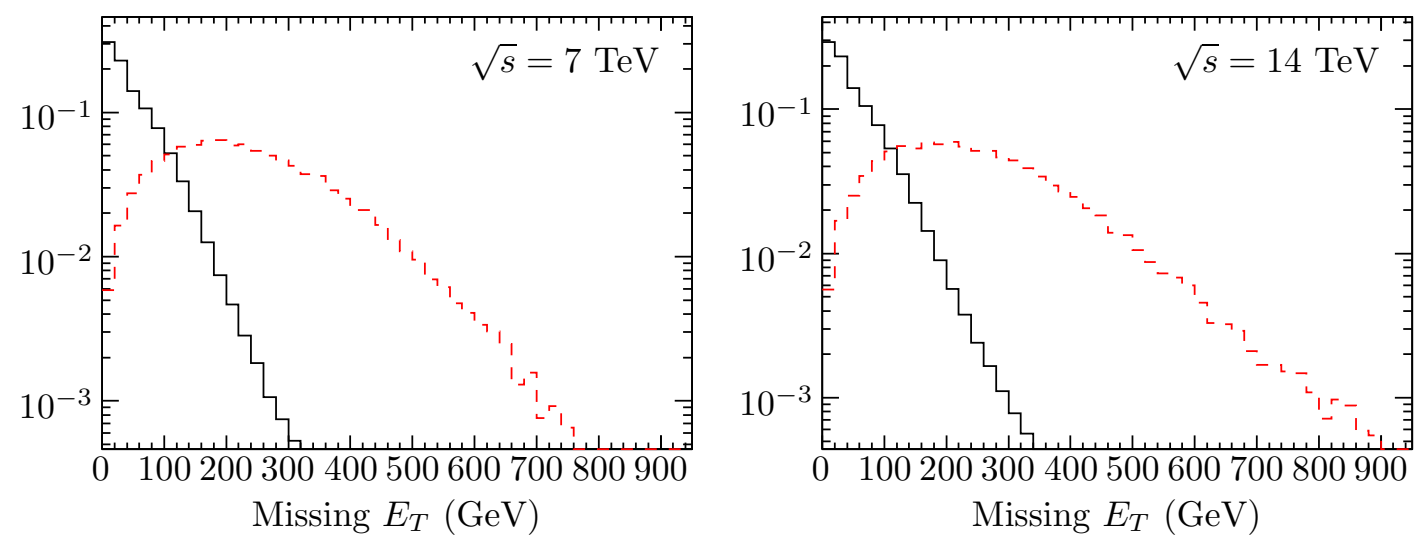

Figure 7. Missing transverse energy at $7 \mathrm{TeV}$ (left) and $14 \mathrm{TeV}$ (right) for the inclusive SUSY sample (dashed) and SM $t \bar{t}$ background (solid). The histograms are normalized to unity.
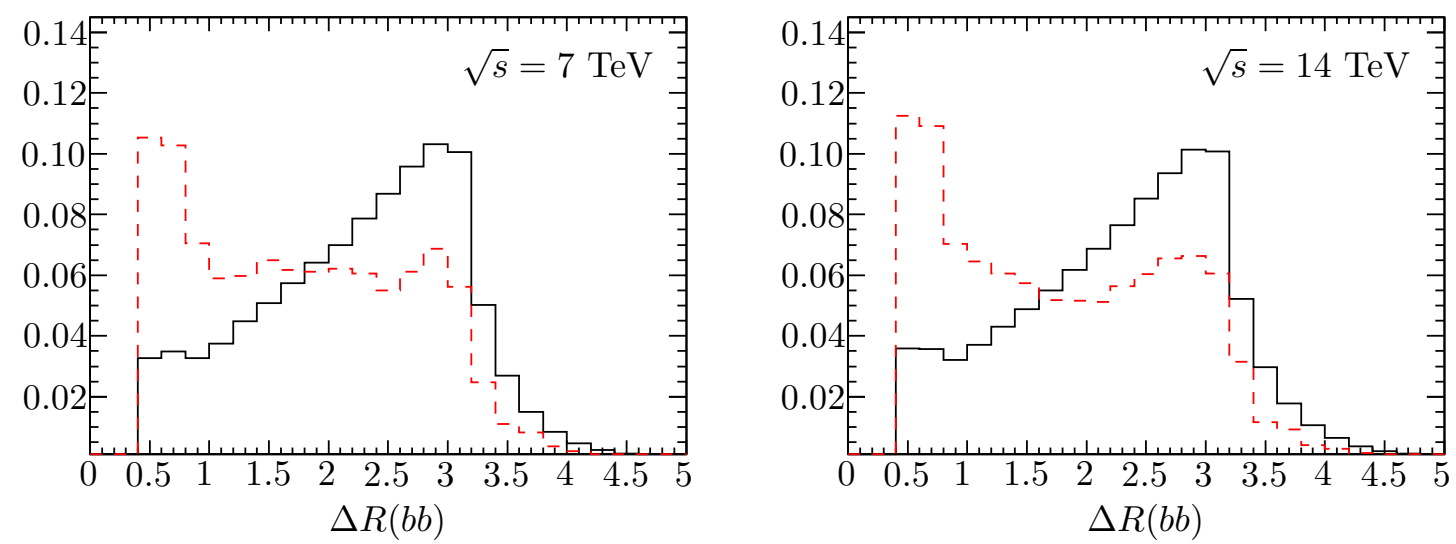

Figure 8. Distribution in $\Delta R(b \bar{b})$ at $7 \mathrm{TeV}$ (left) and $14 \mathrm{TeV}$ (right) for the inclusive SUSY sample (dashed) and SM $t \bar{t}$ background (solid). The histograms are normalized to unity. In events containing more than two $b$-jets, all possible combinations have been included.

mentioned, a hard cut on $\mathbb{E}_{T}$ is also necessary to suppress the background from ordinary QCD multijet events and direct production of $b \bar{b}$. A further advantage of the large $\mathbb{E}_{T}$ is that it can be used for triggering.

The final kinematical distribution we are going to consider is displayed in figure 8. It shows the separation in $\Delta R=\sqrt{(\Delta \eta)^{2}+(\Delta \phi)^{2}}$ between pairs of $b$-jets. For events with $n_{b}>2$ all possible combinations have been included. The signal distribution is seen to peak near the minimum separation of $\Delta R=0.4$ set by the jet measure, while the $t \bar{t}$ background prefers the $b$-jets to be more back-to-back and peaks at $\Delta R \sim \pi$.

The precise cuts applied - and their effect on the event selection - are shown for the SUSY events in table 4 (for the $7 \mathrm{TeV}$ case) and table $5(14 \mathrm{TeV})$. Table 6 gives the corresponding information for the SM $t \bar{t}$ background. Note that the number of generated events in these tables does not correspond to any particular luminosity, but is rather selected to give adequate statistics for the event selection. The inclusive SUSY sample is split into signal and background, where the signal consists of the events containing at least 


\begin{tabular}{|lrrr|}
\hline$M_{\text {SUSY }}=750 \mathrm{GeV}$ & Incl. SUSY & Signal & Background \\
\hline Generated events & 18465 & 8261 & 10195 \\
$n_{j} \geq 2, n_{b} \geq 2$ & 4048 & 2786 & 1262 \\
$p_{T}^{\text {jet } 1}>250 \mathrm{GeV}, p_{T}^{\text {jet} 2}>100 \mathrm{GeV}$ & 2436 & 1738 & 698 \\
$\mathbb{E}_{T}>150 \mathrm{GeV}$ & 1735 & 1211 & 524 \\
$\min [\Delta R(b b)]<1.5$ & 1014 & 774 & 240 \\
\hline Total efficiency & $5.5 \times 10^{-2}$ & $9.4 \times 10^{-2}$ & $2.4 \times 10^{-2}$ \\
\hline \hline$M_{\text {SUSY }}=1 \mathrm{TeV}$ & Incl. SUSY & Signal & Background \\
\hline Generated events & 20671 & 10923 & 9748 \\
$n_{j} \geq 2, n_{b} \geq 2$ & 5313 & 4344 & 969 \\
$p_{T}^{\text {jet } 1}>250 \mathrm{GeV}, p_{T}^{\text {jet } 2}>100 \mathrm{GeV}$ & 4642 & 3828 & 814 \\
$\mathbb{E}_{T}>150 \mathrm{GeV}$ & 3705 & 3036 & 669 \\
min $[\Delta R(b b)]<1.5$ & 2544 & 2170 & 374 \\
\hline Total efficiency & 0.12 & 0.20 & $3.8 \times 10^{-2}$ \\
\hline
\end{tabular}

Table 4. Number of events remaining after each step of the event selection at $\sqrt{s}=7 \mathrm{TeV}$. The SUSY events are classified as signal or background based on the presence of (at least one) Higgs boson in the decay chain. The total number of generated events in the inclusive sample is arbitrary.

one Higgs boson (as determined from Monte Carlo truth information). In the last row we give the accumulated total efficiencies of all the cuts. Looking first at table 4, we see that an efficiency of $5.5 \times 10^{-2}$ is obtained for the case with $M_{\text {SUSY }}=750 \mathrm{GeV}$. This efficiency is more than doubled $(0.12)$ for the case with $M_{\mathrm{SUSY}}=1 \mathrm{TeV}$, since the heavier squarks give harder jets as decay products which leads to more events passing the jet $p_{T}$ cuts. The larger boost given to the LSP at the end of the decay chain also leads to an increased $\mathbb{E}_{T}$. The same qualitative features are visible at $14 \mathrm{TeV}$, as can be read off table 5 . Due to the favorable signal statistics at $14 \mathrm{TeV},{ }^{7}$ we can afford slightly harder cuts on $\mathbb{E}_{T}$ and $\Delta R(b b)$ in this case, something which is also needed to maintain a good background suppression. One should therefore not be discouraged by the somewhat lower efficiencies recorded in this case $\left(4.2 \times 10^{-2}\right.$ for $M_{\mathrm{SUSY}}=750 \mathrm{GeV}$ vs. $9.8 \times 10^{-2}$ for $\left.M_{\mathrm{SUSY}}=1 \mathrm{TeV}\right)$. The signal efficiencies can be compared to those for the $t \bar{t}$ background, given in table 6 , which are at the $10^{-5}$ level for both energies. It is clear from this table that the hard cuts on the jet $p_{T}$ and the $\mathbb{E}_{T}$ distribution are the most important handles available to suppress the background.

As discussed in the previous section, we do not apply any specific cuts to suppress the background from SUSY events that do not involve a Higgs boson. The numbers given in tables 4 and 5 show that nevertheless our event selection gives rise to an improvement also in the ratio of signal events over SUSY-background events. The largest difference in selection efficiency between the SUSY signal and background arises from the typical number of $b$ quarks produced in the two cases, which is larger for the events where Higgs bosons are

\footnotetext{
${ }^{7}$ Going from $7 \mathrm{TeV}$ to $14 \mathrm{TeV}$, the signal cross section for $M_{\mathrm{SUSY}}=750 \mathrm{GeV}$ increases by a factor 14.5 , while the $t \bar{t}$ cross section is only increased by a factor 5 .
} 


\begin{tabular}{|lrrr|}
\hline$M_{\text {SUSY }}=750 \mathrm{GeV}$ & Incl. SUSY & Signal & Background \\
\hline Generated events & 23771 & 10874 & 12897 \\
$n_{j} \geq 2, n_{b} \geq 2$ & 5009 & 3610 & 1399 \\
$p_{T}^{\text {jet1 }}>250 \mathrm{GeV}, p_{T}^{\text {jet} 2}>100 \mathrm{GeV}$ & 3287 & 2422 & 865 \\
$\mathbb{E}_{T}>200 \mathrm{GeV}$ & 1935 & 1400 & 535 \\
min $[\Delta R(b b)]<1.2$ & 991 & 775 & 216 \\
\hline Total efficiency & $4.2 \times 10^{-2}$ & $7.1 \times 10^{-2}$ & $1.7 \times 10^{-2}$ \\
\hline \hline$M_{\text {SUSY }}=1 \mathrm{TeV}$ & Incl. SUSY & Signal & Background \\
\hline Generated events & 20232 & 10557 & 9675 \\
$n_{j} \geq 2, n_{b} \geq 2$ & 5428 & 4338 & 1090 \\
$p_{T}^{\text {jet1 }}>250 \mathrm{GeV}, p_{T}^{\text {jet} 2}>100 \mathrm{GeV}$ & 4852 & 3924 & 928 \\
$\mathbb{E}_{T}>200 \mathrm{GeV}$ & 3392 & 2719 & 673 \\
min $[\Delta R(b b)]<1.2$ & 1983 & 1673 & 310 \\
\hline Total efficiency & $9.8 \times 10^{-2}$ & 0.16 & $3.2 \times 10^{-2}$ \\
\hline
\end{tabular}

Table 5. Events remaining after each step of the event selection at $\sqrt{s}=14 \mathrm{TeV}$. The event categories are similar to those in table 4 . The total number of generated events in the inclusive sample is arbitrary.

\begin{tabular}{|lrr|}
\hline SM $t \bar{t}$ background & $7 \mathrm{TeV}$ & $14 \mathrm{TeV}$ \\
\hline Generated events & 900000 & 2000000 \\
$n_{j} \geq 2, n_{b} \geq 2$ & 259110 & 576232 \\
$p_{T}^{\text {jet } 1}>250 \mathrm{GeV}, p_{T}^{\text {jet} 2}>100 \mathrm{GeV}$ & 1120 & 5189 \\
$\mathbb{E}_{T}>150 \mathrm{GeV}(7 \mathrm{TeV}),>200 \mathrm{GeV}(14 \mathrm{TeV})$ & 102 & 405 \\
$\min [\Delta R(b b)]<1.5(7 \mathrm{TeV}),<1.2(14 \mathrm{TeV})$ & 12 & 61 \\
\hline Total efficiency & $1 \times 10^{-5}$ & $3 \times 10^{-5}$ \\
\hline
\end{tabular}

Table 6. SM $t \bar{t}$ background events remaining after each step of the event selection at $\sqrt{s}=7 \mathrm{TeV}$ and $\sqrt{s}=14 \mathrm{TeV}$. The total number of generated events is arbitrary.

produced, leading to a stronger reduction of the SUSY background by the jet multiplicity cut. The cut on $\Delta R$ also contributes to the difference. This cut has the pleasant "side effect" to enrich the SUSY sample in Higgs events since the jets resulting from $H_{1} \rightarrow b \bar{b}$ decays are more likely to show up for small $\Delta R$ than those from two unpaired $b$-jets.

\subsection{Results}

Figure 9 shows the resulting $b \bar{b}$ mass spectra after final event selection for an integrated luminosity of $5 \mathrm{fb}^{-1}$ at $7 \mathrm{TeV}$. For events with $n_{b}>2$ only the $b$-jet combination minimizing $\Delta R(b b)$ has been included. This reduces effects of combinatorics and increases the sensitivity for discovering resonances in the low mass region. For the scenario with relatively light squarks $\left(M_{\mathrm{SUSY}}=750 \mathrm{GeV}\right)$ shown in the left plot, we observe two peaks close to the masses of the $Z$ boson and $H_{1}$, respectively. There is also a continuous distribution with a tail towards much higher values for $M_{b \bar{b}}$. This results from false pairings, fake $b$-jets, or from $b$-jet pairs of non-resonant origin such as $t$ or $\tilde{b}$ decays. The same qualitative features 

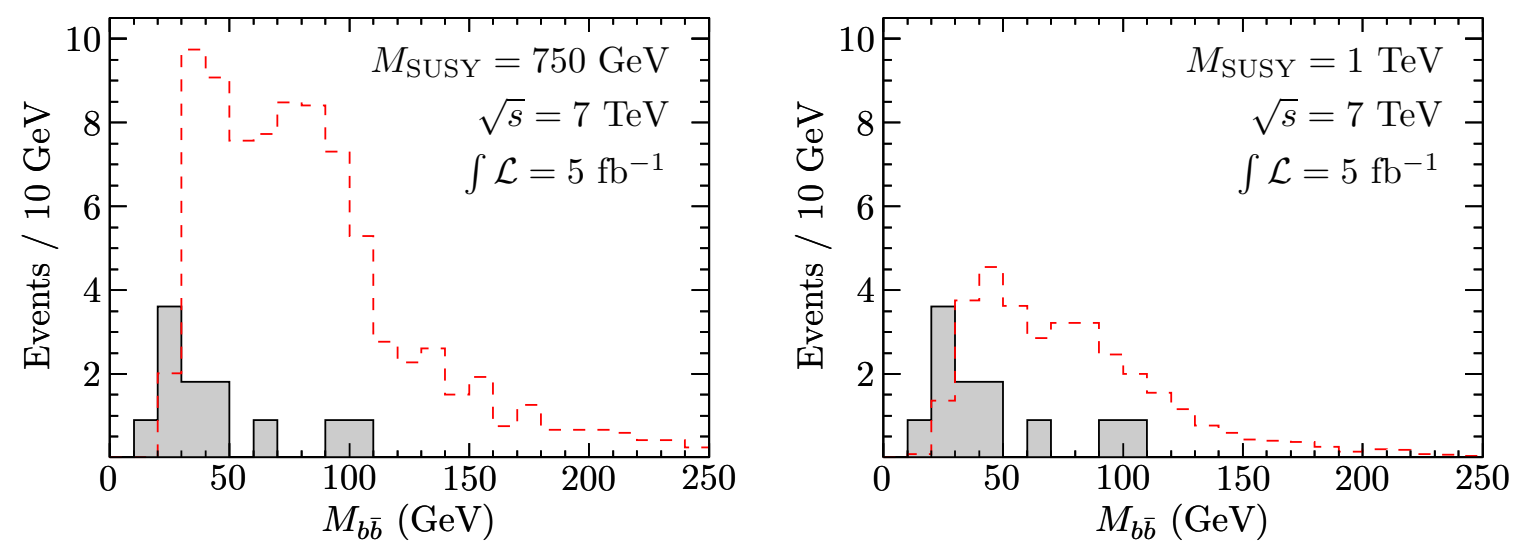

Figure 9. Invariant mass of $b$-jet pairs after final event selection for the inclusive SUSY sample (red, dashed) and SM $t \bar{t}$ background (solid) at $7 \mathrm{TeV}$ in the modified P4 scenario with $M_{\mathrm{SUSY}}=750 \mathrm{GeV}$ (left) and $M_{\mathrm{SUSY}}=1 \mathrm{TeV}$ (right). The histograms have been normalized to an integrated luminosity of $5 \mathrm{fb}^{-1}$.
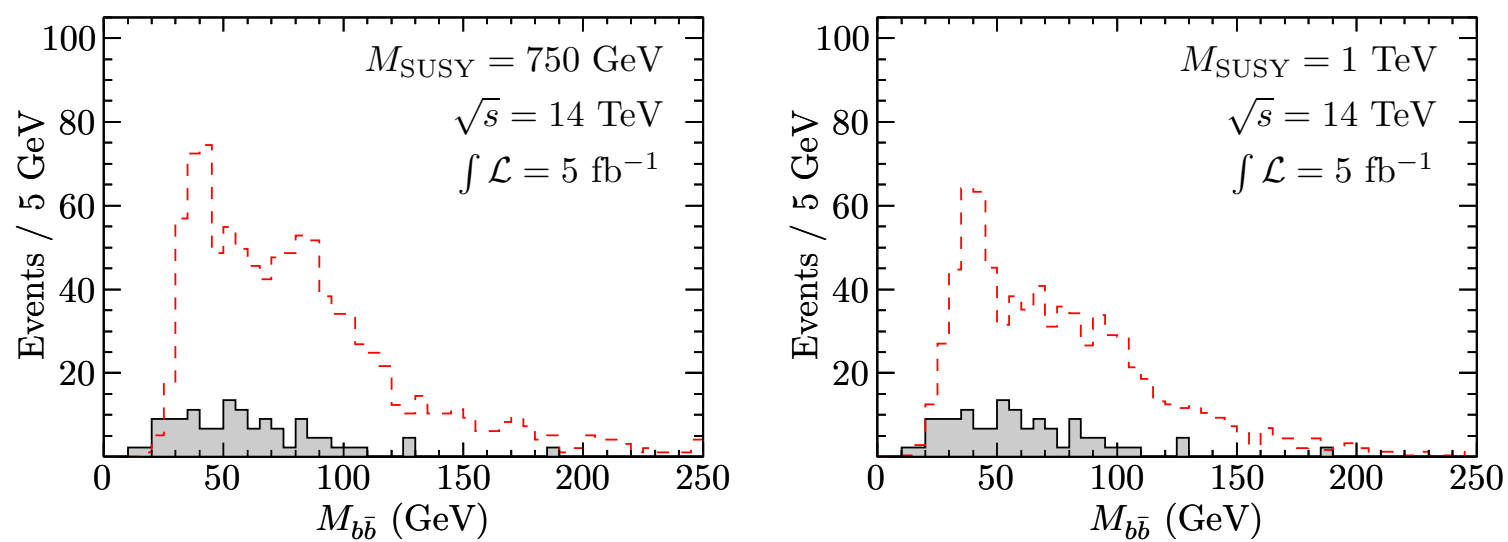

Figure 10. Invariant mass of $b$-jet pairs after final event selection for the inclusive SUSY sample (red, dashed) and SM $t \bar{t}$ background (solid) at $14 \mathrm{TeV}$ in the modified P 4 scenario with $M_{\mathrm{SUSY}}=$ $750 \mathrm{GeV}$ (left) and $M_{\mathrm{SUSY}}=1 \mathrm{TeV}$ (right). The histograms have been normalized to an integrated luminosity of $5 \mathrm{fb}^{-1}$.

are visible in the signal for $M_{\mathrm{SUSY}}=1 \mathrm{TeV}$ (right plot), but the statistics is rather poor due to the low signal cross section. In figure 10 we show the $M_{b \bar{b}}$ distribution at $14 \mathrm{TeV}$, again for an integrated luminosity of $5 \mathrm{fb}^{-1}$. Here the signal statistics is much higher, so that a clear distinction of the $H_{1}$ resonance from the background should be possible both for $M_{\mathrm{SUSY}}=750 \mathrm{GeV}$ (left) and $M_{\mathrm{SUSY}}=1 \mathrm{TeV}$ (right).

The same distributions are shown in figures 11 (for the $\mathrm{LHC}$ at $7 \mathrm{TeV}$ ) and 12 (for $14 \mathrm{TeV}$ ), but here with stacked histograms to more closely resemble "real" data. Here we have furthermore split up the inclusive SUSY sample into signal events (displayed in red), characterised by the presence of (at least) one Higgs boson in the decay chain, and the remaining SUSY background events (black). The latter constitutes an additional source of background besides the SM $t \bar{t}$ background (light gray). In figure 11 we see that the most striking feature is the $H_{1}$ peak. Although the $t \bar{t}$ background peaks at roughly the same 

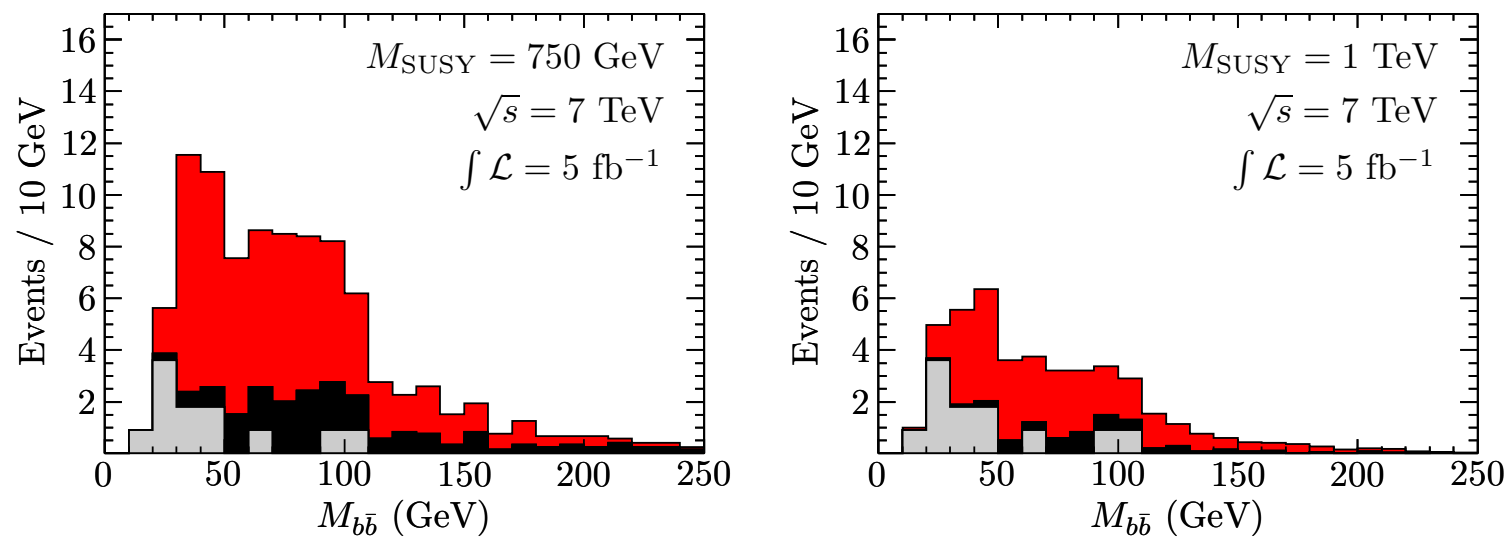

Figure 11. Invariant mass of $b$-jet pairs for SUSY signal (red), SUSY background (black) and $\mathrm{SM} t \bar{t}$ background (light gray) in the modified P4 scenario with $M_{\mathrm{SUSY}}=750 \mathrm{GeV}$ (left) and $M_{\mathrm{SUSY}}=1 \mathrm{TeV}$ (right) at $7 \mathrm{TeV}$ for an integrated luminosity of $5 \mathrm{fb}^{-1}$.
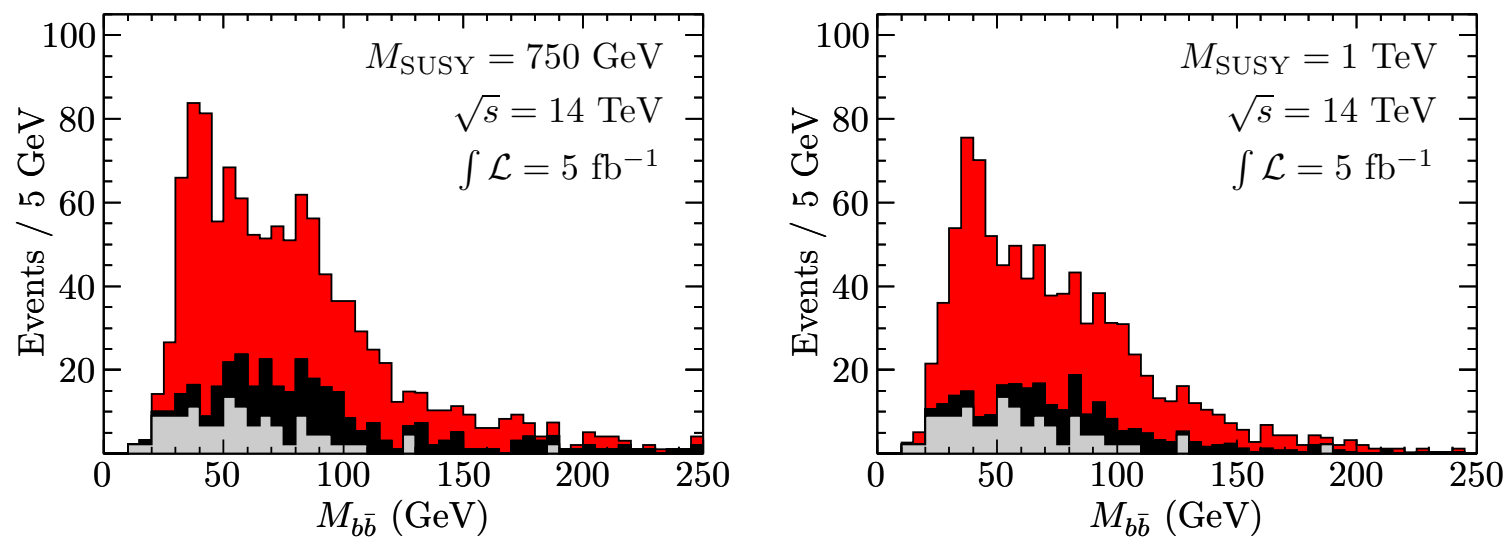

Figure 12. Invariant mass of $b$-jet pairs for SUSY signal (red), SUSY background (black) and SM $t \bar{t}$ background (light gray) in the modified P4 scenario with $M_{\mathrm{SUSY}}=750 \mathrm{GeV}$ (left) and $M_{\mathrm{SUSY}}=1 \mathrm{TeV}$ (right) at $14 \mathrm{TeV}$ for an integrated luminosity of $5 \mathrm{fb}^{-1}$.

position as the signal, the statistics of signal events should be sufficient for establishing a signal over the background. In the $14 \mathrm{TeV}$ case, figure 12 illustrates the features observed already in figure 10 . The $H_{1}$ peak stands out clearly above the background distribution, both for $M_{\mathrm{SUSY}}=750 \mathrm{GeV}$ and $M_{\mathrm{SUSY}}=1 \mathrm{TeV}$.

With the assumed statistics, no peaks are observed in the $b \bar{b}$ mass spectrum for the heavier Higgs bosons $H_{2}$ and $A_{1}$. This is mainly due to the smallness of the branching ratios into the $b \bar{b}$ mode because of the open Higgs decay channels. Part of the difficulty in observing the heavier resonances is also a result of selecting the combination minimizing $\Delta R(b b)$ in configurations with multiple $b$-jets, which favors selection of the light $H_{1}$.

In order to estimate of the significance of the $H_{1}$ mass peak we perform a simple counting of the number of signal (S) and background (B) events in the peak region. For this purpose we perform a Gaussian fit to the maximum of the (full) distributions in figures 11 and 12. Table 7 lists the results extracted from the fit for the mean value $M_{H}$ 


\begin{tabular}{|cccccc|}
\hline$\sqrt{s}$ & $M_{\text {SUSY }}(\mathrm{GeV})$ & $M_{H}(\mathrm{GeV})$ & $\Delta M_{H}(\mathrm{GeV})$ & $S / B$ & $S / \sqrt{B}$ \\
\hline $7 \mathrm{TeV}$ & 750 & 41.6 & 12.2 & 3.4 & 8.2 \\
$7 \mathrm{TeV}$ & 1000 & 37.7 & 17.6 & 1.4 & 3.9 \\
$14 \mathrm{TeV}$ & 750 & 39.5 & 8.0 & 4.5 & 29.7 \\
$14 \mathrm{TeV}$ & 1000 & 39.4 & 9.7 & 4.3 & 29.3 \\
\hline
\end{tabular}

Table 7. Mean value $M_{H}$ and width $\Delta M_{H}$ extracted from a Gaussian fit to the $b \bar{b}$ mass peak. The corresponding number of signal $(S)$ and background $(B)$ events is recorded in the $\pm \Delta M_{H}$ interval around the fitted resonance mass. All results are presented for an integrated luminosity of $5 \mathrm{fb}^{-1}$.

and the $1 \sigma$ width $\Delta M_{H}$ of the peak. We find that the fitted central values reproduce well the correct $H_{1}$ mass for all cases (recall that the input mass used in our numerical simulation is $M_{H_{1}}=40 \mathrm{GeV}$ ). The statistical uncertainty on the mean value $M_{H}$ from the fit is about $\pm 3 \mathrm{GeV}$ for the LHC energy of $7 \mathrm{TeV}$ and about $\pm 1 \mathrm{GeV}$ for $14 \mathrm{TeV}$. This reflects the lower signal statistics available in the low energy running, and the more coarse binning in $M_{b \bar{b}}$ required to observe the peak.

The number of signal and background events in the peak are counted inside an interval $\left[M_{H}-\Delta M_{H}, M_{H}+\Delta M_{H}\right]$, corresponding to $\pm 1 \sigma$ of the Gaussian distribution. As explained above, the combined background includes both the events from SM $t \bar{t}$ and the part of the inclusive SUSY sample containing no Higgs bosons in the cascades. The event numbers are combined into the ratios of signal/background $(S / B)$ and $S / \sqrt{B}$ given in table 7. We use $S / \sqrt{B}$ as a simple illustration for the expected significance and in particular for comparing between the four example cases we consider here and with other theoretical studies using the same criterion. Clearly, claiming an actual discovery would require a more sophisticated statistical treatment. We regard it nevertheless as encouraging that a significance of $S / \sqrt{B}>5$ is achieved for three of the four cases considered in table 7 . The only exception is the case $M_{\mathrm{SUSY}}=1 \mathrm{TeV}$ for the LHC at $7 \mathrm{TeV}$, where $S / \sqrt{B}=3.9$ with $5 \mathrm{fb}^{-1}$. A somewhat higher luminosity (or a combination of ATLAS and CMS data) would be needed in this case in order to reach a significance $S / \sqrt{B} \geq 5$. The very high significances of about 30 obtained for the $14 \mathrm{TeV}$ case illustrate the qualitative features already observed in the discussion of figures 10 and 12: there should be no problems in establishing a signal in this mass region.

\section{$5 \quad$ Summary and conclusions}

The NMSSM is both theoretically appealing as an extension of the SM and interesting phenomenologically, as its spectrum may contain Higgs bosons with mass much below the limits in the SM or the MSSM. We have investigated an NMSSM scenario with a light CP-even Higgs in the mass range $20 \mathrm{GeV}<M_{H_{1}}<M_{Z}$. Scenarios like this may be missed with the standard Higgs search channels at the LHC, in particular due to a potentially large branching ratio of the heavier $\mathrm{H}_{2}$ state, that has SM-like couplings to gauge bosons, into a pair of light Higgses. We have pointed out that there are good prospects for discovering such a light Higgs boson in SUSY cascade decays at the LHC. 
We have performed a Monte Carlo simulation of the signal and the dominant background to the level of fast detector simulation, taking into account also background from other SUSY events that do not involve cascade decays containing a Higgs boson. For our numerical analysis we adapted the "P4" benchmark point proposed for the NMSSM, choosing $M_{H_{1}}=40 \mathrm{GeV}$ as example value for the mass of the light Higgs. Production of squarks and gluinos via the strong interaction at the LHC may give rise to cascade decays involving heavy neutralinos and charginos decaying into lighter ones and a light Higgs. We have investigated the impact of various kinematical variables on discriminating between the inclusive SUSY signal (including events both with and without a Higgs boson in the cascade) and the SM background from $t \bar{t}$ production. A set of simple cuts has been devised that turned out to be efficient for establishing the inclusive SUSY signal. We did not assume any specific knowledge about the background from SUSY events without a Higgs in the cascades. Accordingly, besides favoring events containing the light $H_{1}$ by selecting the combination minimizing $\Delta R(b b)$ in configurations with multiple $b$-jets, we have not applied any particular cuts for suppressing the SUSY background.

Our results show that reconstruction of the decay of the light Higgs into $b \bar{b}$ may be feasible. Such an observation would be a direct experimental sign of the bottom Yukawa coupling, which is difficult to access in standard search channels. We have investigated two values of the soft SUSY-breaking parameter in the squark sector, $M_{\mathrm{SUSY}}=750 \mathrm{GeV}$ and $M_{\mathrm{SUSY}}=1 \mathrm{TeV}$, while we set the gluino mass parameter to $1 \mathrm{TeV}$. A modest integrated luminosity of $5 \mathrm{fb}^{-1}$ has been considered for LHC running both at $7 \mathrm{TeV}$ and $14 \mathrm{TeV}$. We find a statistical significance for the $H_{1}$ mass peak of $S / \sqrt{B} \approx 4$ for $M_{\mathrm{SUSY}}=1 \mathrm{TeV}$ at $\sqrt{s}=7 \mathrm{TeV}$. This significance increases to $S / \sqrt{B} \approx 8$ for $M_{\mathrm{SUSY}}=750 \mathrm{GeV}$ at $7 \mathrm{TeV}$ and reaches a level of almost 30 for both values of $M_{\mathrm{SUSY}}$ at $14 \mathrm{TeV}$. While the example values that we have chosen for $M_{\mathrm{SUSY}}$ and the gluino mass are close to the current search limits from the LHC, the large statistical significance that we have found for the $14 \mathrm{TeV}$ case indicates that there is certainly scope to extend our analysis to scenarios with heavier squarks and gluinos or to scenarios with reduced branching ratios of the neutralinos into Higgs bosons. Since the high-energy run of the LHC is not imminent, we leave a more detailed analysis of this reach for future work.

The results presented here have been obtained in a specific benchmark scenario, but it is easy to see that they are more generally applicable. First of all, the value $M_{H_{1}}=40 \mathrm{GeV}$ used in our numerical analysis was chosen just for illustration. Our results are rather insensitive to the precise value of $M_{H_{1}}$. Since the production relies on the decay of heavier SUSY states, with branching ratios largely independent of $M_{H_{1}}$, the Higgs production rates remain similar for the whole mass range $M_{H_{1}}<M_{Z}$. The event selection and signal identification through $H_{1} \rightarrow b \bar{b}$ proceeds along similar lines as we have discussed.

Concerning the settings of the other SUSY parameters, our results will be similar for other scenarios fulfilling a few simple criteria: Obviously, the neutralinos and charginos have to be sufficiently lighter than the squarks and gluinos in order to be produced at all in the cascade decays of the latter. The squark decays also provide the hard jets utilized in the event selection. With the present limits from the LHC searches on the masses of the gluino and the squarks of the first two generations this criterion is almost automatically 
fulfilled for any model of interest. Furthermore, the neutralino and chargino mass hierarchy and mixing character must be such that the squark decays go through heavier neutralinos or charginos, and the decays of the latter into a light Higgs and a lighter neutralino or chargino are open. Such a scenario is disfavored if the LSP is gaugino-like. In order to generate a sufficient number of Higgs bosons in the cascade decays, it is also advantageous for (at least one of) the gauginos to be heavier than the Higgsinos, so that an intermediate Higgsino decay step can be present. In the NMSSM such a situation can be realized quite easily if the LSP is singlino-like.

While the results presented in this paper are based on a rather simple-minded analysis, involving for instance just a fast detector simulation, we nevertheless regard them as very encouraging, motivating a further exploration of the potential for detecting a light non-SM type Higgs in SUSY cascade decays. In fact, there exists the exciting possibility that the discovery of a SUSY signal could go hand in hand with the discovery of one or more Higgs bosons.

\section{Acknowledgments}

We are grateful to B. Fuks and F. Staub for valuable assistance with generating the FeynArts model file used for parts of this work. We also wish to thank T. Plehn, M. Spira, and S. Brensing for illuminating discussions on the QCD corrections to the squark and gluino production cross sections, U. Ellwanger for discussions on SUSY decays and comparisons to NMSDECAY, and A. Nikitenko, A. Raspereza, and M. Schumacher for their input on various experimental aspects of our analysis. Finally we thank R. Benbrik, S. Heinemeyer, M. Gomez-Bock, and L. Zeune for interesting and useful discussions. This work was supported by the Collaborative Research Center SFB676 of the DFG, "Particles, Strings, and the Early Universe".

Open Access. This article is distributed under the terms of the Creative Commons Attribution Noncommercial License which permits any noncommercial use, distribution, and reproduction in any medium, provided the original author(s) and source are credited.

\section{References}

[1] H.P. Nilles, Supersymmetry, supergravity and particle physics, Phys. Rept. 110 (1984) 1 [INSPIRE].

[2] H.E. Haber and G.L. Kane, The search for supersymmetry: probing physics beyond the standard model, Phys. Rept. 117 (1985) 75 [INSPIRE].

[3] U. Ellwanger, C. Hugonie and A.M. Teixeira, The next-to-minimal supersymmetric standard model, Phys. Rept. 496 (2010) 1 [arXiv:0910.1785] [INSPIRE].

[4] M. Maniatis, The next-to-minimal supersymmetric extension of the standard model reviewed, Int. J. Mod. Phys. A 25 (2010) 3505 [arXiv:0906.0777] [InSPIRE].

[5] ALEPH, DELPHI, L3, OPAL and LEP Working Group for Higgs Boson SEARChes collaborations, S. Schael et al., Search for neutral MSSM Higgs bosons at LEP, Eur. Phys. J. C 47 (2006) 547 [hep-ex/0602042] [INSPIRE]. 
[6] Particle Data Group collaboration, K. Nakamura et al., Review of particle physics, J. Phys. G 37 (2010) 075021 [inSPIRE].

[7] K.E. Williams and G. Weiglein, Precise predictions for $h_{a} \rightarrow h_{b} h_{c}$ decays in the complex MSSM, Phys. Lett. B 660 (2008) 217 [arXiv:0710.5320] [inSPIRE].

[8] K.E. Williams, H. Rzehak and G. Weiglein, Higher order corrections to Higgs boson decays in the MSSM with complex parameters, Eur. Phys. J. C 71 (2011) 1669 [arXiv:1103.1335] [INSPIRE].

[9] R. Dermisek and J.F. Gunion, Escaping the large fine tuning and little hierarchy problems in the next to minimal supersymmetric model and $h \rightarrow$ aa decays,

Phys. Rev. Lett. 95 (2005) 041801 [hep-ph/0502105] [INSPIRE].

[10] R. Dermisek and J.F. Gunion, Consistency of LEP event excesses with an $h \rightarrow$ aa decay scenario and low-fine-tuning NMSSM models, Phys. Rev. D 73 (2006) 111701 [hep-ph/0510322] [INSPIRE].

[11] R. Dermisek and J.F. Gunion, The NMSSM close to the R-symmetry limit and naturalness in $h \rightarrow$ aa decays for $m_{a}<2 m_{b}$, Phys. Rev. D 75 (2007) 075019 [hep-ph/0611142] [INSPIRE].

[12] R. Dermisek and J.F. Gunion, The NMSSM solution to the fine-tuning problem, precision electroweak constraints and the largest LEP Higgs event excess,

Phys. Rev. D 76 (2007) 095006 [arXiv: 0705.4387] [INSPIRE].

[13] R. Dermisek and J.F. Gunion, Many light Higgs bosons in the NMSSM, Phys. Rev. D 79 (2009) 055014 [arXiv:0811.3537] [InSPIRE].

[14] R. Dermisek and J.F. Gunion, New constraints on a light CP-odd Higgs boson and related NMSSM ideal Higgs scenarios, Phys. Rev. D 81 (2010) 075003 [arXiv:1002.1971] [INSPIRE].

[15] M.S. Carena, J.R. Ellis, A. Pilaftsis and C. Wagner, CP violating MSSM Higgs bosons in the light of LEP-2, Phys. Lett. B 495 (2000) 155 [hep-ph/0009212] [INSPIRE].

[16] V. Buescher and K. Jakobs, Higgs boson searches at hadron colliders, Int. J. Mod. Phys. A 20 (2005) 2523 [hep-ph/0504099] [inSPIRE].

[17] M. Schumacher, Investigation of the discovery potential for Higgs bosons of the minimal supersymmetric extension of the standard model (MSSM) with ATLAS, hep-ph/0410112 [INSPIRE].

[18] E. Accomando et al., Workshop on CP studies and non-standard Higgs physics, hep-ph/0608079 [INSPIRE].

[19] A. Akeroyd, Searching for a very light Higgs boson at the Tevatron, Phys. Rev. D 68 (2003) 077701 [hep-ph/0306045] [INSPIRE].

[20] D.K. Ghosh, R. Godbole and D. Roy, Probing the CP-violating light neutral Higgs in the charged Higgs decay at the LHC, Phys. Lett. B 628 (2005) 131 [hep-ph/0412193] [INSPIRE].

[21] K. Cheung, J. Song and Q.-S. Yan, Role of $h \rightarrow \eta \eta$ in intermediate-mass Higgs boson searches at the Large Hadron Collider, Phys. Rev. Lett. 99 (2007) 031801 [hep-ph/0703149] [INSPIRE].

[22] M. Carena, T. Han, G.-Y. Huang and C.E. Wagner, Higgs signal for $h \rightarrow$ aa at hadron colliders, JHEP 04 (2008) 092 [arXiv:0712.2466] [INSPIRE]. 
[23] A.C. Fowler and G. Weiglein, Precise predictions for Higgs production in neutralino decays in the complex MSSM, JHEP 01 (2010) 108 [arXiv:0909.5165] [INSPIRE].

[24] P. Draper, T. Liu and C.E. Wagner, Prospects for Higgs searches at the Tevatron and LHC in the MSSM with explicit CP-violation, Phys. Rev. D 81 (2010) 015014 [arXiv:0911.0034] [INSPIRE].

[25] P. Bandyopadhyay, Higgs production in CP-violating supersymmetric cascade decays: probing the 'open hole' at the Large Hadron Collider, JHEP 08 (2011) 016 [arXiv: 1008.3339] [INSPIRE].

[26] P. Bandyopadhyay and K. Huitu, Production of two Higgses at the Large Hadron Collider in CP-violating supersymmetry, arXiv:1106.5108 [INSPIRE].

[27] LeP Working Group for Higgs boson searches, AlePh, DELPHI, L3 and OPAL collaborations, R. Barate et al., Search for the standard model Higgs boson at LEP, Phys. Lett. B 565 (2003) 61 [hep-ex/0306033] [INSPIRE].

[28] CMS collaboration, G. Bayatian et al., CMS technical design report, volume II: physics performance, J. Phys. G 34 (2007) 995 [inSPIRE].

[29] A. Datta, A. Djouadi, M. Guchait and F. Moortgat, Detection of MSSM Higgs bosons from supersymmetric particle cascade decays at the LHC, Nucl. Phys. B 681 (2004) 31 [hep-ph/0303095] [INSPIRE].

[30] K. Huitu et al., Search for Higgs bosons in SUSY cascades in CMS and dark matter with non-universal gaugino masses, Eur. Phys. J. C 58 (2008) 591 [arXiv:0808.3094] [InSPIRE].

[31] S. Gori, P. Schwaller and C.E. Wagner, Search for Higgs bosons in SUSY cascade decays and neutralino dark matter, Phys. Rev. D 83 (2011) 115022 [arXiv:1103.4138] [INSPIRE].

[32] G.D. Kribs, A. Martin, T.S. Roy and M. Spannowsky, Discovering the Higgs boson in new physics events using jet substructure, Phys. Rev. D 81 (2010) 111501 [arXiv:0912.4731] [INSPIRE].

[33] G.D. Kribs, A. Martin, T.S. Roy and M. Spannowsky, Discovering Higgs bosons of the MSSM using jet substructure, Phys. Rev. D 82 (2010) 095012 [arXiv: 1006.1656] [INSPIRE].

[34] S. Choi, D.J. Miller and P. Zerwas, The neutralino sector of the next-to-minimal supersymmetric standard model, Nucl. Phys. B 711 (2005) 83 [hep-ph/0407209] [INSPIRE].

[35] K. Cheung and T.-J. Hou, Light pseudoscalar Higgs boson in neutralino decays in the next-to-minimal supersymmetric standard model, Phys. Lett. B 674 (2009) 54 [arXiv: 0809.1122] [INSPIRE].

[36] S. Kraml and W. Porod, Sfermion decays into singlets and singlinos in the NMSSM, Phys. Lett. B 626 (2005) 175 [hep-ph/0507055] [INSPIRE].

[37] P.Z. Skands et al., SUSY Les Houches Accord: interfacing SUSY spectrum calculators, decay packages and event generators, JHEP 07 (2004) 036 [hep-ph/0311123] [INSPIRE].

[38] B. Allanach et al., SUSY Les Houches Accord 2, Comput. Phys. Commun. 180 (2009) 8 [arXiv: 0801.0045] [INSPIRE].

[39] A. Djouadi et al., Benchmark scenarios for the NMSSM, JHEP 07 (2008) 002 [arXiv:0801.4321] [INSPIRE].

[40] U. Ellwanger, Radiative corrections to the neutral Higgs spectrum in supersymmetry with a gauge singlet, Phys. Lett. B 303 (1993) 271 [hep-ph/9302224] [INSPIRE]. 
[41] T. Elliott, S. King and P. White, Supersymmetric Higgs bosons at the limit, Phys. Lett. B 305 (1993) 71 [hep-ph/9302202] [INSPIRE].

[42] T. Elliott, S. King and P. White, Squark contributions to Higgs boson masses in the next-to-minimal supersymmetric standard model, Phys. Lett. B 314 (1993) 56 [hep-ph/9305282] [INSPIRE].

[43] T. Elliott, S. King and P. White, Radiative corrections to Higgs boson masses in the next-to-minimal supersymmetric standard model, Phys. Rev. D 49 (1994) 2435 [hep-ph/9308309] [INSPIRE].

[44] P. Pandita, One loop radiative corrections to the lightest Higgs scalar mass in nonminimal supersymmetric standard model, Phys. Lett. B 318 (1993) 338 [INSPIRE].

[45] U. Ellwanger and C. Hugonie, Yukawa induced radiative corrections to the lightest Higgs boson mass in the NMSSM, Phys. Lett. B 623 (2005) 93 [hep-ph/0504269] [INSPIRE].

[46] G. Degrassi and P. Slavich, On the radiative corrections to the neutral Higgs boson masses in the NMSSM, Nucl. Phys. B 825 (2010) 119 [arXiv:0907.4682] [INSPIRE].

[47] U. Ellwanger, J.F. Gunion and C. Hugonie, NMHDECAY: a Fortran code for the Higgs masses, couplings and decay widths in the NMSSM, JHEP 02 (2005) 066 [hep-ph/0406215] [INSPIRE].

[48] U. Ellwanger and C. Hugonie, NMHDECAY 2.0: an updated program for sparticle masses, Higgs masses, couplings and decay widths in the NMSSM,

Comput. Phys. Commun. 175 (2006) 290 [hep-ph/0508022] [INSPIRE].

[49] G. Bélanger, F. Boudjema, C. Hugonie, A. Pukhov and A. Semenov, Relic density of dark matter in the NMSSM, JCAP 09 (2005) 001 [hep-ph/0505142] [INSPIRE].

[50] F. Mahmoudi, J. Rathsman, O. Stål and L. Zeune, Light Higgs bosons in phenomenological NMSSM, Eur. Phys. J. C 71 (2011) 1608 [arXiv:1012.4490] [INSPIRE].

[51] D. Eriksson, F. Mahmoudi and O. Stål, Charged Higgs bosons in minimal supersymmetry: updated constraints and experimental prospects, JHEP 11 (2008) 035 [arXiv:0808.3551] [INSPIRE].

[52] D. Stöckinger, The muon magnetic moment and supersymmetry, J. Phys. G 34 (2007) R45 [hep-ph/0609168] [INSPIRE].

[53] U. Ellwanger, Enhanced di-photon Higgs signal in the next-to-minimal supersymmetric standard model, Phys. Lett. B 698 (2011) 293 [arXiv:1012.1201] [INSPIRE].

[54] U. Ellwanger, J.F. Gunion and C. Hugonie, Establishing a no lose theorem for NMSSM Higgs boson discovery at the LHC, hep-ph/0111179 [INSPIRE].

[55] U. Ellwanger, J.F. Gunion, C. Hugonie and S. Moretti, Towards a no lose theorem for NMSSM Higgs discovery at the LHC, hep-ph/0305109 [INSPIRE].

[56] U. Ellwanger, J.F. Gunion, C. Hugonie and S. Moretti, NMSSM Higgs discovery at the LHC, hep-ph/0401228 [INSPIRE].

[57] J.R. Forshaw, J.F. Gunion, L. Hodgkinson, A. Papaefstathiou and A.D. Pilkington, Reinstating the 'no-lose' theorem for NMSSM Higgs discovery at the LHC, JHEP 04 (2008) 090 [arXiv:0712.3510] [INSPIRE].

[58] A. Belyaev et al., The scope of the 4 tau channel in Higgs-strahlung and vector boson fusion for the NMSSM no-lose theorem at the LHC, arXiv:0805.3505 [INSPIRE]. 
[59] U. Ellwanger, Higgs bosons in the next-to-minimal supersymmetric standard model at the LHC, Eur. Phys. J. C 71 (2011) 1782 [arXiv:1108.0157] [InSPIRE].

[60] V. Barger, P. Langacker and G. Shaughnessy, Neutralino signatures of the singlet extended MSSM, Phys. Lett. B 644 (2007) 361 [hep-ph/0609068] [INSPIRE].

[61] S. Liebler and W. Porod, Electroweak corrections to neutralino and chargino decays into a W-boson in the (N)MSSM, Nucl. Phys. B 849 (2011) 213 [arXiv:1011.6163] [InSPIRE].

[62] T. Hahn, Generating Feynman diagrams and amplitudes with FeynArts 3, Comput. Phys. Commun. 140 (2001) 418 [hep-ph/0012260] [InSPIRE].

[63] T. Hahn and M. Pérez-Victoria, Automatized one loop calculations in four-dimensions and D-dimensions, Comput. Phys. Commun. 118 (1999) 153 [hep-ph/9807565] [InSPIRE].

[64] N.D. Christensen and C. Duhr, FeynRules - Feynman rules made easy, Comput. Phys. Commun. 180 (2009) 1614 [arXiv:0806.4194] [INSPIRE].

[65] C. Duhr and B. Fuks, A superspace module for the FeynRules package, Comput. Phys. Commun. 182 (2011) 2404 [arXiv:1102.4191] [INSPIRE].

[66] F. Staub, From superpotential to model files for FeynArts and CalcHep/CompHEP, Comput. Phys. Commun. 181 (2010) 1077 [arXiv:0909.2863] [INSPIRE].

[67] D. Das, U. Ellwanger and A.M. Teixeira, NMSDECAY: a Fortran code for supersymmetric particle decays in the next-to-minimal supersymmetric standard model, arXiv:1106.5633 [INSPIRE].

[68] W. Beenakker, R. Hopker, M. Spira and P.M. Zerwas, Squark and gluino production at hadron colliders, Nucl. Phys. B 492 (1997) 51 [hep-ph/9610490] [INSPIRE].

[69] J. Pumplin, New generation of parton distributions with uncertainties from global QCD analysis, JHEP 07 (2002) 012 [hep-ph/0201195] [INSPIRE].

[70] W. Beenakker, M. Krämer, T. Plehn, M. Spira and P.M. Zerwas, Stop production at hadron colliders, Nucl. Phys. B 515 (1998) 3 [hep-ph/9710451] [INSPIRE].

[71] ATLAS collaboration, G. Aad et al., Search for supersymmetry using final states with one lepton, jets and missing transverse momentum with the ATLAS detector in $\sqrt{s}=7 \mathrm{TeV} p$, Phys. Rev. Lett. 106 (2011) 131802 [arXiv:1102.2357] [INSPIRE].

[72] ATLAS collaboration, G. Aad et al., Search for squarks and gluinos using final states with jets and missing transverse momentum with the ATLAS detector in $\sqrt{s}=7 \mathrm{TeV}$ proton-proton collisions, Phys. Lett. B 701 (2011) 186 [arXiv:1102.5290] [INSPIRE].

[73] ATLAS collaboration, G. Aad et al., Search for supersymmetry in pp collisions at $\sqrt{s}=7 \mathrm{TeV}$ in final states with missing transverse momentum and b-jets, Phys. Lett. B 701 (2011) 398 [arXiv:1103.4344] [INSPIRE].

[74] ATLAS collaboration, G. Aad et al., Search for an excess of events with an identical flavour lepton pair and significant missing transverse momentum in $\sqrt{s}=7 \mathrm{TeV}$ proton-proton collisions with the ATLAS detector, Eur. Phys. J. C 71 (2011) 1647 [arXiv:1103.6208] [INSPIRE].

[75] ATLAS collaboration, G. Aad et al., Search for supersymmetric particles in events with lepton pairs and large missing transverse momentum in $\sqrt{s}=7$ TeV proton-proton collisions with the ATLAS experiment, Eur. Phys. J. C 71 (2011) 1682 [arXiv:1103.6214] [INSPIRE]. 
[76] CMS collaboration, V. Khachatryan et al., Search for supersymmetry in pp collisions at $7 \mathrm{TeV}$ in events with jets and missing transverse energy, Phys. Lett. B 698 (2011) 196 [arXiv:1101.1628] [INSPIRE].

[77] CMS collaboration, S. Chatrchyan et al., Search for neutral MSSM Higgs bosons decaying to tau pairs in pp collisions at $\sqrt{s}=7$ TeV, Phys. Rev. Lett. 106 (2011) 231801 [arXiv:1104.1619] [INSPIRE].

[78] CMS collaboration, S. Chatrchyan et al., Search for supersymmetry in events with a lepton, a photon and large missing transverse energy in pp collisions at $\sqrt{s}=7 \mathrm{Te} V$, JHEP 06 (2011) 093 [arXiv:1105.3152] [INSPIRE].

[79] CMS collaboration, S. Chatrchyan et al., Search for supersymmetry in events with $b$ jets and missing transverse momentum at the LHC, JHEP 07 (2011) 113 [arXiv:1106.3272] [INSPIRE].

[80] ATLAS collaboration, Search for squarks and gluinos using final states with jets and missing transverse momentum with the ATLAS detector in sqrts $=7$ TeV proton-proton collisions, ATLAS-CONF-2011-086.

[81] CMS collaboration, Search for supersymmetry in all-hadronic events with $\alpha_{T}$, PAS-SUS-11-003.

[82] J. Alwall et al., MadGraph/MadEvent v4: the new web generation, JHEP 09 (2007) 028 [arXiv:0706.2334] [INSPIRE].

[83] T. Sjöstrand, S. Mrenna and P.Z. Skands, PYTHIA 6.4 physics and manual, JHEP 05 (2006) 026 [hep-ph/0603175] [INSPIRE].

[84] S. Ovyn, X. Rouby and V. Lemaitre, DELPHES, a framework for fast simulation of a generic collider experiment, arXiv:0903.2225 [INSPIRE].

[85] M. Cacciari, G.P. Salam and G. Soyez, The anti- $k_{t}$ jet clustering algorithm, JHEP 04 (2008) 063 [arXiv:0802.1189] [InSPIRE].

[86] ATLAS collaboration, G. Aad et al., Expected performance of the ATLAS experiment detector, trigger and physics, arXiv:0901.0512 [INSPIRE].

[87] M. Aliev et al., HATHOR: HAdronic Top and Heavy quarks crOss section calculatoR, Comput. Phys. Commun. 182 (2011) 1034 [arXiv:1007.1327] [INSPIRE].

[88] A.D. Martin, W.J. Stirling, R.S. Thorne and G. Watt, Parton distributions for the LHC, Eur. Phys. J. C 63 (2009) 189 [arXiv:0901.0002] [INSPIRE]. 